\title{
COMBABLE GROUPS HAVE GROUP COHOMOLOGY OF POLYNOMIAL GROWTH
}

\author{
RALF MEYER
}

\begin{abstract}
Group cohomology of polynomial growth is defined for any finitely generated discrete group, using cochains that have polynomial growth with respect to the word length function. We give a geometric condition that guarantees that it agrees with the usual group cohomology and verify this condition for a class of combable groups. Our condition involves a chain complex that is closely related to exotic cohomology theories studied by Allcock and Gersten and by Mineyev.
\end{abstract}

\section{INTRODUCTION}

Let $G$ be a finitely generated discrete group and let $\ell$ be a word length function on $G$. In a standard complex that computes the group cohomology of $G$, one can define a subcomplex by allowing only cochains of polynomial growth with respect to $\ell$ in each variable. The cohomology of this subcomplex is the group cohomology of polynomial growth of $G$, which we denote by $H_{\mathrm{pol}}^{n}(G)$. We may ask whether the canonical maps $H_{\mathrm{pol}}^{n}(G) \rightarrow H^{n}(G)$ are isomorphisms. This question came up in the work of Alain Connes and Henri Moscovici on the Novikov conjecture for hyperbolic groups in 3 and has also been studied by Ronghui Ji in [8].

We define a certain chain complex of bornological vector spaces $\mathscr{S} \tilde{C}_{\bullet}(G)$ and prove the following two facts. First, $\mathscr{S} \tilde{C} \bullet(G)$ has a bounded contracting homotopy if the group $G$ is combable in the sense of [4]. Secondly, the existence of such a contracting homotopy implies $H_{\mathrm{pol}}^{n}(G) \cong H^{n}(G)$. An important feature of our construction is that the homotopy type of the chain complex $\mathscr{S} \tilde{C} \bullet(G)$ is a quasiisometry invariant of $G$. In contrast, the problem of whether $H_{\mathrm{pol}}^{n}(G) \cong H^{n}(G)$ does not seem invariant under quasi-isometry.

The chain complex $\mathscr{S} \tilde{C}_{\bullet}(G)$ is related to the convolution algebra

$$
\mathscr{S}(G):=\left\{f: G \rightarrow \mathbb{R}\left|\sum_{g \in G}\right| f(g) \mid(\ell(g)+1)^{k}<\infty \quad \forall k \in \mathbb{N}\right\} .
$$

If we use the Banach algebra $\ell_{1}(G)$ instead, we obtain a complex that is homotopy equivalent to one constructed by Daniel J. Allcock and Stephen M. Gersten in [1] (for groups for which the classifying space $B G$ has finite type). However, already the chain complex $\ell_{1} \tilde{C} \cdot(\mathbb{Z})$ has non-trivial homology and hence cannot be contractible. The best one can say is that the range of the differential in this complex is dense in the kernel, that is, the "reduced homology" vanishes. Igor Mineyev shows in 13 that this happens for groups with a sufficiently nice combing. However, this seems too weak to compare the bounded cohomology and the usual group cohomology.

We now explain the definition of $\mathscr{S} \tilde{C}_{\bullet}(G)$. For any set $X$, let $S(X)$ be the simplicial set whose $n$-simplices are the $n+1$-tuples $\left(x_{0}, \ldots, x_{n}\right) \in X^{n+1}$ and whose $j$ th face and degeneracy maps leave out and double $x_{j}$, respectively. Let $C \bullet(X)$ be

2000 Mathematics Subject Classification. 20F32, 20J05, 20 J06.

This research was supported by the EU-Network Quantum Spaces and Noncommutative Geometry (Contract HPRN-CT-2002-00280) and the Deutsche Forschungsgemeinschaft (SFB 478). 
the reduced simplicial chain complex associated to $S(X)$ with coefficients $\mathbb{R}$. We identify $C_{n}(X)$ with the space of functions $X^{n+1} \rightarrow \mathbb{R}$ with finite support that vanish on $\left(x_{0}, \ldots, x_{n}\right)$ if $x_{j}=x_{j+1}$ for some $j \in\{0, \ldots, n-1\}$. We define the augmentation map $\alpha: C_{0}(X) \rightarrow \mathbb{R}$ on basis vectors by $x \mapsto 1$ for all $x \in X$. We define a subcomplex $\tilde{C}_{\bullet}(X)$ by $\tilde{C}_{0}(X)=\operatorname{ker} \alpha$ and $\tilde{C}_{n}(X)=C_{n}(X)$ for $n \geq 1$.

Any map $f: X \rightarrow Y$ induces a chain map $f_{*}: \tilde{C}_{\bullet}(X) \rightarrow \tilde{C}_{\bullet}(Y)$, and there is an explicit chain homotopy between $f_{*}$ and $f_{*}^{\prime}$ for any $f, f^{\prime}: X \rightarrow Y$. Hence $\tilde{C}_{\bullet}(X)$ is always contractible because it is homotopy equivalent to $\tilde{C}_{\bullet}(\star)=0$.

If $(X, d)$ is a discrete proper metric space, we let $\mathscr{S} C_{n}(X)$ be the space of functions $f: X^{n+1} \rightarrow \mathbb{R}$ with the following properties:

(1) there is $R>0$ such that $f\left(x_{0}, \ldots, x_{n}\right)=0$ if $d\left(x_{i}, x_{j}\right) \geq R$ for some $i, j \in$ $\{0, \ldots, n\}$, or if $x_{j}=x_{j+1}$ for some $j \in\{0, \ldots, n-1\}$;

(2) for all $k \in \mathbb{N}$ and all fixed $\star \in X$, we have

$$
\sum_{x_{0}, \ldots, x_{n} \in X}\left|f\left(x_{0}, \ldots, x_{n}\right)\right| \cdot\left(d\left(x_{0}, \star\right)+\cdots+d\left(x_{n}, \star\right)+1\right)^{k}<\infty .
$$

The space $\mathscr{S} C_{n}(X)$ is a bornological vector space that contains $C_{n}(X)$ as a dense subspace. The differential of $C_{\bullet}(X)$ may be extended and turns $\mathscr{S} C_{\bullet}(X)$ into a chain complex of bornological vector spaces. Finally, $\mathscr{S} \tilde{C}_{\bullet}(X)$ is the kernel of the augmentation map $\mathscr{S} C_{\bullet}(X) \rightarrow \mathbb{R}$.

A combing on a metric space $(X, d)$ with a chosen base point $\star$ is a sequence of maps $f_{n}: X \rightarrow X$ with the following properties: $f_{0}(x)=\star$ for all $x \in X$, and for any $x \in X$ there exists $n \in \mathbb{N}$ such that $f_{N}(x)=x$ for all $N \geq n$; the maps $f_{n}$ are uniformly quasi-Lipschitz; and the pairs of maps $\left(f_{n}, f_{n+1}\right)$ are uniformly close in the sense that the set of $d\left(f_{n}(x), f_{n+1}(x)\right)$ for $x \in X, n \in \mathbb{N}$ is bounded. We say that the combing has polynomial growth if the number of $n \in \mathbb{N}$ with $f_{n}(x) \neq f_{n+1}(x)$ is controlled by a polynomial in $d(x, \star)$. Such combings exist for all hyperbolic groups, for automatic groups, and for groups that are combable in the sense of [4]. However, it seems that such combings do not exist for non-Abelian nilpotent groups.

Our first main result is that $\mathscr{S} \tilde{C}_{\bullet}(X)$ has a bounded contracting homotopy if $X$ has a combing of polynomial growth. The proof is actually quite simple. Since the maps $f_{n}, f_{n+1}$ are close for each $n \in \mathbb{N}$, there is an explicit chain homotopy $H\left(f_{n}, f_{n+1}\right): \mathscr{S} \tilde{C}_{\bullet}(X) \rightarrow \mathscr{S} \tilde{C} \bullet(X)$ between the maps induced by $f_{n}$ and $f_{n+1}$. The sum $H=\sum_{n \in \mathbb{N}} H\left(f_{n}, f_{n+1}\right)$ is the desired contracting homotopy. The hypotheses on $\left(f_{n}\right)$ guarantee that $H$ is a bounded linear operator on $\mathscr{S} \tilde{C}_{\bullet}(X)$.

Our second main result is that $H_{\text {pol }}^{n}(G) \cong H^{n}(G)$ if $\mathscr{S} \tilde{C}_{\bullet}(G)$ has a bounded contracting homotopy. This requires some homological algebra with bornological modules over the convolution algebras $\mathbb{R}[G]$ and $\mathscr{S}(G)$. These two are bornological unital algebras, and the embedding $i: \mathbb{R}[G] \rightarrow \mathscr{S}(G)$ is a bounded unital algebra homomorphism. We work with bornologies instead of topologies because this gives better results for spaces that are built out of $\mathbb{R}[G]$ and $\mathscr{S}(G)$. The right category of modules over a bornological unital algebra $A$ (like $\mathbb{R}[G]$ or $\mathscr{S}(G)$ ) is the category $\operatorname{Mod}(A)$ of bornological left $A$-modules. Such a module is defined by a bounded unital algebra homomorphism $A \rightarrow \operatorname{Hom}(M, M)$ or, equivalently, a bounded bilinear map $A \times M \rightarrow M$ satisfying the usual properties. The morphisms are the bounded $A$-linear maps. Homological algebra in $\operatorname{Mod}(A)$ works as usual if we only admit extensions with a bounded linear section and resolutions with a bounded linear contracting homotopy. This is explained in detail in [10].

In order to keep this article accessible for readers with a background in geometric group theory, we only use the most basic homological algebra and do not explore all consequences of the contractibility of $\mathscr{S} \tilde{C} \bullet(G)$. This is done in [10], where I also 
show by other techniques that $\mathscr{S} \tilde{C}_{\bullet}(G)$ is contractible for groups of polynomial growth. This improves the main result of 8 .

Let $\hat{\otimes}$ be the projective complete bornological tensor product $([6])$. A bornological $A$-module of the form $A \hat{\otimes} X$ with the obvious module structure is called free. Free modules are projective for extensions with a bounded linear section. The usual argument in homological algebra that shows that two free resolutions of the same module are homotopy equivalent still works in our setting.

The group cohomology of $G$ can be defined as $H^{n}(G) \cong \operatorname{Ext}_{\mathbb{R}[G]}^{n}(\mathbb{R}, \mathbb{R})$, where $\mathbb{R}$ is equipped with the trivial representation of $G$ and the resulting module structure over $\mathbb{R}[G]$. That is, it is the cohomology of the chain complex $\operatorname{Hom}_{\mathbb{R}[G]}\left(P_{\bullet}, \mathbb{R}\right)$, where $P_{\bullet} \rightarrow \mathbb{R}$ is some free $\mathbb{R}[G]$-module resolution of $\mathbb{R}$. If we let $G$ act diagonally on $C_{n}(G)$, then the chain complex $C_{\bullet}(G)$ constructed above becomes such a free $\mathbb{R}[G]$-module resolution. Similarly, the group cohomology of polynomial growth is isomorphic to the cohomology $\operatorname{Ext}_{\mathscr{S}(G)}^{n}(\mathbb{R}, \mathbb{R})$ of the chain complex $\operatorname{Hom}_{\mathscr{S}(G)}\left(P_{\bullet}^{\prime}, \mathbb{R}\right)$, where $P_{\bullet}^{\prime} \rightarrow \mathbb{R}$ is some free $\mathscr{S}(G)$-module resolution of $\mathbb{R}$.

Let $i^{*}: \operatorname{Mod}(\mathscr{S}(G)) \rightarrow \operatorname{Mod}(\mathbb{R}[G])$ be the functor induced by the embedding $i: \mathbb{R}[G] \rightarrow \mathscr{S}(G)$. We also define a functor

$$
i_{!}: \operatorname{Mod}(\mathscr{S}(G)) \rightarrow \operatorname{Mod}(\mathbb{R}[G]), \quad i_{!}(M) \cong \mathscr{S}(G) \hat{\otimes}_{\mathbb{R}[G]} M .
$$

These functors are adjoint, that is,

$$
\operatorname{Hom}_{\mathscr{S}(G)}\left(i_{!}(M), N\right) \cong \operatorname{Hom}_{\mathbb{R}[G]}\left(M, i^{*}(N)\right)
$$

if $M$ and $N$ are bornological left modules over $\mathbb{R}[G]$ and $\mathscr{S}(G)$, respectively. Moreover, $i_{!}\left(C_{\bullet}(G)\right) \cong \mathscr{S} C_{\bullet}(G)$. If $\mathscr{S} \tilde{C}_{\bullet}(G)$ has a bounded contracting homotopy, then $i_{!}(C \bullet(G))$ is a free $\mathscr{S}(G)$-module resolution of $\mathbb{R}$. Hence we may use it to compute $\operatorname{Ext}_{\mathscr{S}(G)}^{n}(\mathbb{R}, \mathbb{R})$. Since $i^{*}(\mathbb{R})=\mathbb{R}$, the adjointness relation $(1)$ yields

$$
\begin{aligned}
& \operatorname{Ext}_{\mathscr{S}(G)}^{n}(\mathbb{R}, \mathbb{R}) \cong H^{n}\left(\operatorname{Hom}_{\mathscr{S}(G)}\left(i_{!}(C \bullet(G)), \mathbb{R}\right)\right) \\
& \cong H^{n}\left(\operatorname{Hom}_{\mathbb{R}[G]}\left(C_{\bullet}(G), \mathbb{R}\right)\right) \cong \operatorname{Ext}_{\mathbb{R}[G]}^{n}(\mathbb{R}, \mathbb{R}) .
\end{aligned}
$$

We now summarise the following sections. In Section 2 we define bornological vector spaces $\mathscr{S}(X)=\mathscr{S}^{\infty}(X), \mathscr{S}^{\omega}(X)$ and $\mathscr{O}(X)$ of functions of polynomial, subexponential, and exponential decay. Along the way, we recall some basic facts about bornologies because they went out of fashion a few decades ago. Let $\mathscr{T}(X)$ be one of the function spaces mentioned above. In Section 3 we define a chain complex $C_{\bullet}(X)$ for any set $X$ and enlarge it to $\mathscr{T} C \bullet(X)$ if $X$ is a discrete proper metric space. We check that $\mathscr{T} C_{\bullet}(X)$ is functorial for quasi-Lipschitz maps and that close maps induce chain homotopic chain maps. In Section 4 we explain the notion of combing that we use and prove that $\mathscr{T} C \bullet(X)$ is contractible if $X$ has a combing of appropriate growth.

In Section 5, we define group cohomology with polynomial, subexponential, and exponential growth and identify these theories with extension groups in the bornological module categories of $\mathscr{S}^{\infty}(G), \mathscr{S}^{\omega}(G)$, and $\mathscr{O}(G)$, respectively. Then we prove that the tempered group cohomology associated to $\mathscr{T}(G)$ is isomorphic to the usual group cohomology if the reduced chain complex $\mathscr{T} \tilde{C}_{\bullet}(G)$ has a bounded contracting homotopy. In addition, we prove that $H^{n}(G)$ is finite-dimensional for all $n \in \mathbb{N}$ if $\mathscr{T} \tilde{C}_{\bullet}(G)$ is contractible. Hence $\mathscr{S} \tilde{C}_{\bullet}(G)$ may fail to be contractible.

It is desirable to replace $\mathscr{T} C \bullet(X)$ by smaller and more geometric chain complexes. We discuss how to do this in Sections 6 and 7 . If $X$ is a group, then $\mathscr{T} C \bullet(X)$ is homotopy equivalent to $i_{!}\left(P_{\bullet}\right)$ for any free $\mathbb{R}[G]$-module resolution of $\mathbb{R}$. This allows to study the case of non-Abelian free groups in great detail. One particular way to construct free $\mathbb{R}[G]$-module resolutions of the trivial representation is to use the cellular chain complex Cell•( $Y$ ) of a contractible $\mathrm{CW}$-complex on which $G$ acts 
properly by cellular maps. We investigate what additional structure on $Y$ is needed to write down the tempered versions of Cell• $(Y)$. This yields a sufficient condition for $\mathscr{T}$ Cellø $(Y) \cong \mathscr{T} C \bullet(X)$ that works if $X$ is just a proper discrete metric space.

The results of this article were announced at a conference at the Mathematische Forschungsinstitut Oberwolfach in 2004 (see [11). More recently, I have applied similar techniques to the Schwartz algebras of reductive $p$-adic groups ([12]).

I would like to thank Heath Emerson for helpful discussions about coarse geometry and geometric group theory.

\section{FUnCTION SPACES ON DisCRETE METRIC SPACES}

Let $X$ be a discrete, proper metric space. Here and in the following, properness means that bounded subsets of $X$ are finite. Let $\mathbb{R}[X]$ be the free $\mathbb{R}$-vector space on the set $X$; that is, $\mathbb{R}[X]$ is a vector space containing $X$ as a basis. We often identify $\mathbb{R}[X]$ with the space of functions $X \rightarrow \mathbb{R}$ of finite support. Fix any point $\star \in X$ and define $\ell(x):=d(x, \star)$. Define norms on $\mathbb{R}[X]$ by

$$
\|\phi\|^{k}:=\sum_{x \in X}|\phi(x)|(\ell(x)+1)^{k}
$$

for any $k \in \mathbb{R}$. We let $\mathscr{S}^{k}(X)$ be the Banach space completion of $\mathbb{R}[X]$ for this norm. Equivalently, $\mathscr{S}^{k}(X)$ consists of those functions $\phi: X \rightarrow \mathbb{R}$ with $\|\phi\|^{k}<\infty$. We get equivalent norms if we change $\star$ or replace $d$ by a metric that is quasi-isometric to it (that is, the identity map $(X, d) \rightarrow\left(X, d^{\prime}\right)$ is a quasi-isometry). Hence such modifications yield equivalent spaces $\mathscr{S}^{k}(X)$. Let $\mathscr{S}(X)$ be the space of functions $\phi: X \rightarrow \mathbb{R}$ that satisfy $\|\phi\|^{k}<\infty$ for all $k \in \mathbb{N}$. This is a Fréchet space with respect to the topology that is defined by the sequence of norms $\left\|_{\mathcal{L}}\right\|^{k}, k \in \mathbb{N}$. Later we shall usually treat $\mathscr{S}(X)$ as a bornological vector space.

A bornology on a vector space is a collection of subsets, called bounded subsets, satisfying the following axioms (see $\sqrt{7}$ ): subsets of bounded subsets are again bounded; finite subsets are bounded; if $S_{1}, S_{2}$ are bounded, so is $S_{1}+S_{2}$; if $S$ is bounded, so is $\bigcup_{|t| \leq \lambda} t \cdot S$ for any $\lambda \in \mathbb{R}$. Two important examples are the families of von Neumann bounded subsets and of precompact subsets of a Fréchet space. We call these bornologies the von Neumann bornology and the precompact bornology. Recall that a subset of a Fréchet space $V$ is called von Neumann bounded if it is bounded with respect to all continuous seminorms. Since $V$ is complete, there is no difference between relatively compact and precompact subsets. By the way, "precompact" is a synonym for "totally bounded". Usually, the von Neumann bornology is easier to describe explicitly than the precompact bornology, but the precompact bornology may have better analytical properties.

One checks easily that the embedding $\mathscr{S}^{k+1}(X) \rightarrow \mathscr{S}^{k}(X)$ is a compact map for all $k \in \mathbb{N}$. By definition, this means that $\mathscr{S}(X)$ is a Fréchet-Schwartz space. In a Fréchet-Schwartz space, any von Neumann bounded subset is relatively compact, that is, the von Neumann bornology and the precompact bornology agree (see [7]). We always equip $\mathscr{S}(X)$ with this bornology. We may equip the Banach spaces $\mathscr{S}^{k}(X)$ with the precompact bornology or the von Neumann bornology. This makes no difference for the following constructions.

In this article all bornologies are complete and convex. This means that for any bounded subset $S \subseteq V$ there exists a subspace $V_{T} \subseteq V$ together with a Banach space norm on $V_{T}$ such that the embedding $V_{T} \rightarrow V$ is a bounded linear map and such that $S$ is a bounded subset in $V_{T}$. The Banach space norm on $V_{T}$ can be described equally well by its closed unit ball. This is a subset $T \subseteq V$, which we call a complete disk in $V$. The complete disks in any complete bornological vector space $V$ form a directed set. The space $V$ is canonically isomorphic to the direct 
limit of the associated inductive system of Banach spaces $\left(V_{T}\right)$. Various concepts of functional analysis are defined in the bornological context by reduction to these Banach spaces. For instance, a sequence in $V$ converges if and only if it converges in $V_{T}$ for some complete disk $T$. Since we only use complete convex bornologies in the following, we drop these qualifiers from our notation and agree that bornology, henceforth, stands for complete convex bornology.

Another important example of a bornology is the fine bornology, which is defined for any vector space $V$. It consists of those subsets that are contained in and bounded in some finite-dimensional subspace of $V$. It is the finest (that is, smallest) possible bornology on $V$. This is a reasonable bornology if $V$ is a vector space like $\mathbb{R}[X]$. Analysis in fine bornological vector spaces is quite easy because it always reduces to finite-dimensional subspaces. In contrast, the finest locally convex topology on a space like $\mathbb{R}[X]$ is rather unwieldy. The bornological and topological ways of doing analysis turn out to be equivalent for Fréchet spaces like $\mathscr{S}(X)$ in many situations (see $[9]$ ). For spaces like $\mathbb{R}[X]$ there are some technical differences, where the bornological approach gives better results.

A linear map $f: V \rightarrow W$ between two bornological vector spaces is called bounded if it maps bounded subsets of $V$ to bounded subsets of $W$. The bounded linear maps are the morphisms in the category of bornological vector spaces. If $V$ and $W$ are both Fréchet spaces equipped with the von Neumann or precompact bornology, then a linear map $V \rightarrow W$ is bounded if and only if it is continuous. If $V$ carries the fine bornology and $W$ is arbitrary, then any linear map $V \rightarrow W$ is bounded.

We frequently have to express the fact that some bornological vector space $V$, say, $V=\mathbb{R}[X]$ is contained in another bornological vector space $W$, say, $W=\mathscr{S}(X)$, such that the embedding $V \rightarrow W$ is a bounded linear map with dense range. We simply write $V \precsim W$ to express all of the above. "Dense range" means that a bounded linear map $W \rightarrow X$ into another bornological vector space that vanishes on $V$ already vanishes on all of $W$ (see also [9] for other notions of density).

After this digression into functional analysis, we construct some more function spaces. For any $\alpha>0$, let $\ell_{1}\left(X, \alpha^{\ell}\right):=\left\{\phi: X \rightarrow \mathbb{R} \mid\|\phi\|_{\alpha}<\infty\right\}$, where

$$
\|\phi\|_{\alpha}:=\sum_{x \in X}|\phi(x)| \alpha^{\ell(x)} .
$$

Although $\|\triangleleft\|_{\alpha}$ and $\ell_{1}\left(X, \alpha^{\ell}\right)$ change if we rescale the metric, the spaces

$$
\begin{array}{rlr}
\mathscr{O}(X):=\{\phi: X \rightarrow \mathbb{R} \mid \forall \alpha>1: & \left.\|\phi\|_{\alpha}<\infty\right\} \\
\mathscr{S}^{\omega}(X):=\{\phi: X \rightarrow \mathbb{R} \mid \exists \alpha>1: & \left.\|\phi\|_{\alpha}<\infty\right\}
\end{array}
$$

only depend on the quasi-isometry class of the metric.

The sequence of norms $\left\|_{-}\right\|_{n}$ for $n \in \mathbb{N}$ turns $\mathscr{O}(X)$ into a Fréchet-Schwartz space because the embeddings $\iota_{\alpha, \beta}: \ell_{1}\left(X, \alpha^{\ell}\right) \rightarrow \ell_{1}\left(X, \beta^{\ell}\right)$ for $\alpha>\beta$ are compact. Hence the von Neumann bornology and the precompact bornology on $\mathscr{O}(X)$ agree. We choose this bornology on $\mathscr{O}(X)$. We equip $\mathscr{S}^{\omega}(X)$ with the direct limit bornology. Thus a subset of $\mathscr{S}^{\omega}(X)$ is bounded if and only if it is bounded with respect to the norm $\|\sim\|_{1+1 / n}$ for some $n \in \mathbb{N}$. A bornological vector space that is a direct limit of a sequence of Banach spaces with compact structure maps is called a Silva space (see $[7])$. Thus $\mathscr{S}^{\omega}(X)$ is a Silva space.

There is a duality between Fréchet-Schwartz spaces and Silva spaces, see [7]. The dual of a topological vector space carries a canonical bornology: the equicontinuous bornology, consisting of equicontinuous families of linear functionals. The dual of a Fréchet-Schwartz space with this bornology is a Silva space. The dual of a bornological vector space carries a canonical topology: the topology of uniform 
convergence on bounded subsets. The dual of a Silva space with this topology is a Fréchet-Schwartz space. These two operations are inverse to each other.

We need to know the dual spaces of the function spaces defined above. Since $\mathbb{R}[X]$ is a dense subspace in any of them, a bounded linear functional is determined by its values on the basis vectors $x \in X$ and hence given by $\phi \mapsto \sum_{x \in X} \phi(x) \psi(x)$ for some function $\psi: X \rightarrow \mathbb{R}$. Fix such a function $\psi$. It gives rise to a bounded linear functional on $\mathscr{S}^{k}(X)$ for $k \in \mathbb{N}$ if and only if $\psi$ has polynomial growth of order $k$, that is, $\psi(x) \leq C(\ell(x)+1)^{k}$ for some $C>0, k \in \mathbb{N}$. It gives rise to a bounded linear functional on $\mathscr{S}(X)$ if and only if it has polynomial growth of some order $k \in \mathbb{N}$. It gives rise to a bounded linear functional on $\mathscr{S}^{\omega}(X)$ if and only if it has subexponential growth, that is, for any $\alpha>1$ there is $C>0$ such that $\psi(x) \leq C \cdot \alpha^{\ell(x)}$ for all $x \in X$. A function $\psi$ gives rise to a bounded linear functional on $\mathscr{O}(X)$ if and only if it has exponential growth, that is, $\psi(x) \leq C \cdot \alpha^{\ell(x)}$ for all $x \in X$ for some $C>0, \alpha>1$. All these dual spaces carry canonical bornologies. The spaces of functions of polynomial and exponential growth are Silva spaces, the space of functions of subexponential growth is a Fréchet-Schwartz space.

Let $X$ and $Y$ be discrete proper metric spaces. A map $f: X \rightarrow Y$ is called quasi-Lipschitz or large scale Lipschitz if there is $C>0$ such that $d(f(x), f(y)) \leq$ $C(d(x, y)+1)$ for all $x, y \in X$. Then the linear map $f_{*}: \mathbb{R}[X] \rightarrow \mathbb{R}[Y]$ defined by $f_{*}\left(\delta_{x}\right):=\delta_{f(x)}$ or, equivalently, $f_{*} \phi(y):=\sum_{f(x)=y} \phi(x)$, extends to bounded linear operators $\mathscr{S}^{k}(X) \rightarrow \mathscr{S}^{k}(Y)$ for $k \in \mathbb{R}_{+} \cup\{\infty, \omega\}$ and $\mathscr{O}(X) \rightarrow \mathscr{O}(Y)$. This is a special feature of $\ell_{1}$-norms. For instance, $\ell_{2}(X)$ is only functorial if the number of pre-images of points in $Y$ is bounded. Thus $\ell_{2}(X)$ is not even functorial for quasiisometries.

Now let $G$ be a finitely generated discrete group equipped with a word metric, so that $\ell$ is the word length function associated to some set of generators. Hence $\ell(x y) \leq \ell(x)+\ell(y)$ for all $x, y \in G$. This implies easily that the norms $\left\|_{-}\right\|^{k}$ and $\left\|\_\right\|_{\alpha}$ are submultiplicative. Thus $\mathscr{S}^{k}(G)$ and $\ell_{1}\left(G, \alpha^{\ell}\right)$ are Banach algebras for all $k \in \mathbb{N}, \alpha>1$. It follows that $\mathscr{S}^{\infty}(G)$ and $\mathscr{O}(G)$ are locally multiplicative Fréchet algebras and that $\mathscr{S}^{\omega}(G)$ is a bornological algebra.

\section{TEMPERED FREE CHAIN COMPLEXES}

We first define a chain complex $C \bullet(X)$ for any non-empty set $X$ and discuss its functoriality. Then we modify it using a metric on $X$.

The free simplicial set $S(X)$ over a non-empty set $X$ is the following simplicial set. We let $S_{n}(X)$ be the set of all $n+1$-tuples $\left(x_{0}, \ldots, x_{n}\right) \in X^{n+1}$. The $j$ th face map $S_{n}(X) \rightarrow S_{n-1}(X)$ omits $x_{j}$, the $j$ th degeneracy map $S_{n}(X) \rightarrow S_{n+1}(X)$ doubles $x_{j}$. Let $C_{n}(X)$ be the vector space spanned by the nondegenerate simplices in $S_{n}(X)$. Thus a basis of $C_{n}(X)$ is given by the $n+1$-tuples $\left(x_{0}, \ldots, x_{n}\right)$ with $x_{j} \neq x_{j+1}$ for all $j \in\{0, \ldots, n-1\}$. Equivalently, $C_{n}(X)$ is the space of functions $\phi: X^{n+1} \rightarrow \mathbb{R}$ with finite support that satisfy $\phi\left(x_{0}, \ldots, x_{n}\right)=0$ if $x_{j}=x_{j+1}$ for some $j \in\{0, \ldots, n-1\}$. We equip $C_{n}(X)$ with the fine bornology.

The simplicial boundary map $\delta: C_{n}(X) \rightarrow C_{n-1}(X)$ is given on basis vectors by

$$
\delta\left(x_{0}, \ldots, x_{n}\right):=\sum_{j=0}^{n}(-1)^{j}\left(x_{0}, \ldots, \widehat{x_{j}}, \ldots, x_{n}\right) .
$$

As usual, $\widehat{x_{j}}$ means omission of $x_{j}$. It is easy to check by hand that $\delta$ is well-defined and satisfies $\delta^{2}=0$. In terms of functions, we have

$$
\delta \phi\left(x_{0}, \ldots, x_{n-1}\right)=\sum_{j=0}^{n} \sum_{y \in X}(-1)^{j} \phi\left(x_{0}, \ldots, x_{j-1}, y, x_{j}, \ldots, x_{n-1}\right)
$$


for all $\phi \in C_{n}(X)$. We call $C_{\bullet}(X):=\left(C_{n}(X), \delta\right)_{n \geq 0}$ the free chain complex over $X$.

Any map $f: X \rightarrow Y$ induces a morphism of simplicial sets $S(X) \rightarrow S(Y)$ by

$$
f_{*}\left(x_{0}, \ldots, x_{n}\right):=\left(f\left(x_{0}\right), \ldots, f\left(x_{n}\right)\right) \quad \forall n \in \mathbb{N}, x_{0}, \ldots, x_{n} \in X .
$$

This induces a chain map $f_{*}: C_{\bullet}(X) \rightarrow C_{\bullet}(Y)$ by the same formula.

Let $f, f^{\prime}: X \rightarrow Y$ be two maps. There is an elementary homotopy between the induced maps $f_{*}, f_{*}^{\prime}: S(X) \rightarrow S(Y)$. On the chain complex level, it is given by

$$
\begin{aligned}
H\left(f, f^{\prime}\right): C_{n}(X) & \rightarrow C_{n+1}(Y), \\
\left(x_{0}, \ldots, x_{n}\right) & \mapsto \sum_{j=0}^{n}(-1)^{j}\left(f\left(x_{0}\right), \ldots, f\left(x_{j}\right), f^{\prime}\left(x_{j}\right), \ldots, f^{\prime}\left(x_{n}\right)\right) .
\end{aligned}
$$

Since $x_{j}$ occurs twice, $\left(f\left(x_{0}\right), \ldots, f\left(x_{j}\right), f^{\prime}\left(x_{j}\right), \ldots, f^{\prime}\left(x_{n}\right)\right)$ contains a repetition if $\left(x_{0}, \ldots, x_{n}\right)$ does. Hence $H\left(f, f^{\prime}\right)$ is well-defined. We claim that

$$
H\left(f, f^{\prime}\right) \circ \delta+\delta \circ H\left(f, f^{\prime}\right)=f_{*}^{\prime}-f_{*} .
$$

We verify (2) on a generator $\left(x_{0}, \ldots, x_{n}\right)$. All the summands in $H\left(f, f^{\prime}\right) \delta$ also occur in $\delta H\left(f, f^{\prime}\right)$ with opposite signs and hence cancel out. The only terms in $(-1)^{j} \delta\left(f\left(x_{0}\right), \ldots, f\left(x_{j}\right), f^{\prime}\left(x_{j}\right), \ldots, f^{\prime}\left(x_{n}\right)\right)$ that survive are

$\left(f\left(x_{0}\right), \ldots, f\left(x_{j-1}\right), f^{\prime}\left(x_{j}\right), \ldots, f^{\prime}\left(x_{n}\right)\right)-\left(f\left(x_{0}\right), \ldots, f\left(x_{j}\right), f^{\prime}\left(x_{j+1}\right), \ldots, f^{\prime}\left(x_{n}\right)\right)$.

Summing over $j$, another cancellation occurs and yields (2).

As a consequence, the constant maps $X \rightarrow \star \rightarrow X$ yield a homotopy equivalence between $S(X)$ and $S(\star)$ and hence between $C_{\bullet}(X)$ and $C_{\bullet}(\star)$ for any point $\star \in X$. Evidently, $|S(\star)|=\star$ and $C_{\bullet}(\star)$ is just $\mathbb{R}$ in degree 0 . The homotopy equivalence $C \bullet(X) \rightarrow \mathbb{R}$ is the augmentation map

$$
\alpha: C_{0}(X)=\mathbb{R}[X] \rightarrow \mathbb{R}, \quad \alpha \phi=\sum_{x \in X} \phi(x) .
$$

We let $\tilde{C}_{\bullet}(X) \subseteq C_{\bullet}(X)$ be the subcomplex with $\tilde{C}_{n}(X):=C_{n}(X)$ for $n \geq 1$ and $\tilde{C}_{0}(X):=\operatorname{ker} \alpha \subseteq C_{0}(X)$. Thus $\tilde{C}_{\bullet}(X)$ is contractible for any set $X$.

Now let $X$ be a discrete proper metric space. Let $\mathscr{T}(X)$ be one of the function spaces $\mathscr{S}^{k}(X)$ for $k \in \mathbb{R}_{+} \cup\{\infty, \omega\}$ or $\mathscr{O}(X)$ that we have defined in Section 2 . For any $R \in \mathbb{N}$, we let $\mathscr{T} C_{n}(X)_{R}$ be the space of all functions $\phi: X^{n+1} \rightarrow \mathbb{R}$ that satisfy the following two conditions:

control: $\phi\left(x_{0}, \ldots, x_{n}\right)=0$ if $d\left(x_{i}, x_{j}\right)>R$ for some $i, j \in\{0, \ldots, n\}$ or $x_{j}=x_{j+1}$ for some $j \in\{0, \ldots, n-1\}$;

growth: the function $x \mapsto \sum_{x_{1}, \ldots, x_{n} \in X}\left|\phi\left(x, x_{1}, \ldots, x_{n}\right)\right|$ belongs to $\mathscr{T}(X)$.

A subset of $\mathscr{T} C_{n}(X)_{R}$ is bounded if the set of functions in $\mathscr{T}(X)$ that arises in the second condition is bounded in $\mathscr{T}(X)$.

Since bounded subsets of $X$ are finite, the control condition for functions in $\mathscr{T} C_{n}(X)_{R}$ ensures that the sums in the growth condition are finite. The norms that define $\mathscr{T}(X)$ only depend on $\ell$, and $|\ell(x)-\ell(y)| \leq R$ if $d(x, y) \leq R$. Hence we can rewrite the growth condition more symmetrically. A function $\phi: X^{n+1} \rightarrow \mathbb{R}$ with controlled support belongs to $\mathscr{S}^{k} C_{n}(X)_{R}$ for $k \in \mathbb{R}_{+}$if and only if

$$
\sum_{x_{0}, \ldots, x_{n} \in X}\left|\phi\left(x_{0}, \ldots, x_{n}\right)\right|\left(\ell\left(x_{0}\right)+\cdots+\ell\left(x_{n}\right)+1\right)^{k}<\infty .
$$

We have $\phi \in \mathscr{S}^{\infty} C_{n}(X)_{R}$ if and only if these norms remain finite for all $k \in \mathbb{N}$. We leave it to the reader to formulate similar descriptions for $\mathscr{S}^{\omega}(X)_{R}$ and $\mathscr{O}(X)_{R}$.

We let $\mathscr{T} C_{n}(X)$ be the inductive limit of the subspaces $\mathscr{T} C_{n}(X)_{R}, R \in \mathbb{N}$, equipped with the inductive limit bornology. That is, a subset of $\mathscr{T} C_{n}(X)$ is bounded if it is contained in and bounded in $\mathscr{T} C_{n}(X)_{R}$ for some $R \in \mathbb{N}$. For 
$n=0$ the control condition is empty and we simply get $\mathscr{T} C_{0}(X)=\mathscr{T}(X)$. The augmentation map (3) extends to a bounded linear functional on $\mathscr{T}(X)$. Hence we get a 1-codimensional closed subspace $\mathscr{T} \tilde{C}_{0}(X):=\operatorname{ker} \alpha \subseteq \mathscr{T} C_{0}(X)$. We also let $\mathscr{T} \tilde{C}_{n}(X)=\mathscr{T} C_{n}(X)$ for $n \geq 1$. One checks easily that the boundary map $\delta$ extends to a bounded linear operator $\mathscr{T} \tilde{C}_{n}(X) \rightarrow \mathscr{T} \tilde{C}_{n-1}(X)$ for all $n \geq 1$. Furthermore, we have $\tilde{C}_{n}(X) \precsim \mathscr{T} \tilde{C}_{n}(X)$ and $C_{n}(X) \precsim \mathscr{T} C_{n}(X)$ for all $n \in \mathbb{N}$ because $\mathbb{R}[X] \precsim \mathscr{T}(X)$.

Definition 1. Let $X$ be a discrete proper metric space and let $\mathscr{T}(X)$ be $\mathscr{S}^{k}(X)$ for $k \in \mathbb{R}_{+} \cup\{\infty, \omega\}$ or $\mathscr{O}(X)$. The chain complexes of bornological vector spaces

$$
\mathscr{T} C \bullet(X)=\left(\mathscr{T} C_{n}(X), \delta\right)_{n \geq 0}, \quad \mathscr{T} \tilde{C} \bullet(X)=\left(\mathscr{T} \tilde{C}_{n}(X), \delta\right)_{n \geq 0}
$$

are called $\mathscr{T}(X)$-tempered free chain complex over $X$ and $\mathscr{T}(X)$-tempered reduced free chain complex over $X$, respectively.

Proposition 2. If $X$ and $Y$ are quasi-isometric, then the chain complexes $\mathscr{T} \tilde{C}_{\bullet}(X)$ and $\mathscr{T} \tilde{C}_{\bullet}(Y)$ are bornologically homotopy equivalent. (This means that the chain maps and homotopies involved are bounded.)

Proof. The assertion follows from the following two functoriality assertions. Let $X$ and $Y$ be two proper discrete metric spaces and let $f: X \rightarrow Y$ be a quasi-Lipschitz map. Then the induced chain map $f_{*}: \tilde{C}_{\bullet}(X) \rightarrow \tilde{C}_{\bullet}(Y)$ extends to a bounded linear map $f_{*}: \mathscr{T} \tilde{C}_{\bullet}(X) \rightarrow \mathscr{T} \tilde{C}_{\bullet}(Y)$. Let $f, f^{\prime}: X \rightarrow Y$ be two quasi-Lipschitz maps that are close in the sense that $d\left(f(x), f^{\prime}(x)\right)$ remains bounded. Then $H\left(f, f^{\prime}\right)$ extends to a bounded operator $H\left(f, f^{\prime}\right): \mathscr{T} \tilde{C}_{\bullet}(X) \rightarrow \mathscr{T} \tilde{C}_{\bullet}(Y)$, which still satisfies (2). That is, the maps $f_{*}, f_{*}^{\prime}: \mathscr{T} \tilde{C}_{\bullet}(X) \rightarrow \mathscr{T} \tilde{C}_{\bullet}(Y)$ are bornologically homotopy equivalent.

A constant map $X \rightarrow \star \rightarrow X$, which is of course Lipschitz, induces the zero map on $\mathscr{T} \tilde{C}_{\bullet}(X)$. However, it is not close to the identity map unless $X$ is finite. Hence $\mathscr{T} \tilde{C} \bullet(X)$ has a chance to be non-trivial.

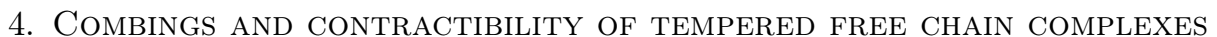

In this section we find a sufficient condition for $\mathscr{T} \tilde{C} \bullet(X)$ to be contractible, based on the notion of a combing of a metric space. This notion is due to William P. Thurston and grew out of the theory of automatic groups developed in [4]. More recently, geometric group theorists have become interested in more general classes of combings than those allowed in $\sqrt{4}$ in order to overcome the following problem: the only nilpotent groups that are combable in the sense of [4] are the virtually Abelian ones. It is possible to find combings with somewhat weaker properties on certain nilpotent groups (see [2,5]). However, these combings are asynchronous, while the argument below needs synchronous combings.

Definition 3. Let $X$ be a discrete proper metric space, choose $\star \in X$. A combing on $X$ is a sequence of maps $f_{n}: X \rightarrow X, n \in \mathbb{N}$, such that

- for all $x \in X$, we have $f_{0}(x)=\star$ and there is $n \in \mathbb{N}$ such that $f_{N}(x)=x$ for all $N \geq n$;

- the maps $\left(f_{n}\right)_{n \in \mathbb{N}}$ are uniformly quasi-Lipschitz, that is, there exists $C>0$ with $d\left(f_{n}(x), f_{n}(y)\right) \leq C \cdot(d(x, y)+1)$ for all $x, y \in X, n \in \mathbb{N}$;

- the pairs of maps $\left(f_{n}, f_{n+1}\right)_{n \in \mathbb{N}}$ are uniformly close in the sense that there is $S \in \mathbb{N}$ such that $d\left(f_{n}(x), f_{n+1}(x)\right) \leq S$ for all $x \in X, n \in \mathbb{N}$.

Let $J(x)$ be the number of $n \in \mathbb{N}$ with $f_{n}(x) \neq f_{n+1}(x)$. Since the sequence $\left(f_{n}(x)\right)$ is eventually constant, $J(x)<\infty$ for all $x \in X$. A combing has polynomial growth of order $m \in \mathbb{N}$ if $J(x) \leq C(\ell(x)+1)^{m}$ for some $C \in \mathbb{R}_{+}$. Subexponential growth and exponential growth are defined similarly. 
The function $J$ is related to the length of a combing in [5]. If $G$ is a finitely generated discrete group, then a combing in the above sense exists on $G$ if and only if $G$ has a synchronous combing in the notation of [5]. The additional conditions that are imposed on combings in [4] imply linear growth. That is, all assertions of Theorem 5 below apply to groups that are combable in the sense of [4]. This class of groups contains all automatic groups and therefore all hyperbolic groups. Thus it contains finitely generated free non-Abelian groups and free groups. The only nilpotent groups that are combable in the sense of [4] are the Abelian ones.

One checks easily that the existence of a combing on $X$ (with specified growth) is independent of the choice of $\star$ and a quasi-isometry invariant.

Example 4. Finitely generated free groups have a combing of linear growth. Let $\mathbb{F}_{r}$ be the free group on $r$ generators, which we denote by $s(1), \ldots, s(r)$. Also let $s(r+j)=s(j)^{-1}$ for $j=1, \ldots, r$. Let $\ell$ be the word length for this set of generators, and let $d(g, h)=\ell\left(g^{-1} h\right)$ be the associated left invariant distance. We define the sequence of maps $\left(f_{n}\right)$ as follows. Write $g \in \mathbb{F}_{r}$ as a reduced word $g=s\left(i_{1}\right) \cdots s\left(i_{\ell}\right)$ in the generators, that is, $\ell=\ell(g)$. Let $f_{n}(g)=s\left(i_{1}\right) \cdots s\left(i_{n}\right)$ for $0 \leq n<\ell$ and $f_{n}(g)=g$ for $n \geq \ell$. Thus $J(g)=\ell(g)$ for this sequence of maps. One checks easily that $d\left(f_{n}(g), f_{n+1}(g)\right) \leq 1$ for all $g \in \mathbb{F}_{r}, n \in \mathbb{N}$, and $d\left(f_{n}(g), f_{n}(h)\right) \leq d(g, h)$ for all $g, h \in \mathbb{F}_{r}, n \in \mathbb{N}$. Hence we have a combing of linear growth.

Theorem 5. Let $X$ be a discrete proper metric space. If $X$ has a combing of polynomial growth of order $m \in \mathbb{N}$, then the embeddings $\mathscr{S}^{k+m} \tilde{C}_{\bullet}(X) \rightarrow \mathscr{S}^{k} \tilde{C}_{\bullet}(X)$ are homotopic to zero for all $k \in \mathbb{R}_{+}$.

If $X$ has a combing of polynomial growth, then $\mathscr{S}^{\infty} \tilde{C}_{\bullet}(X)$ is contractible.

If $X$ has a combing of subexponential growth, then $\mathscr{S}^{\omega} \tilde{C} \bullet(X)$ is contractible.

If $X$ has a combing of exponential growth, then $\mathscr{O} \tilde{C}_{\bullet}(X)$ is contractible.

Proof. We only prove the first assertion; the others are proven similarly. The definition of $H\left(f, f^{\prime}\right)$ shows that $H\left(f, f^{\prime}\right)\left(x_{0}, \ldots, x_{n}\right)=0$ unless $f\left(x_{j}\right) \neq f^{\prime}\left(x_{j}\right)$ for some $j \in\{0, \ldots, n\}$. Therefore, for a basis vector $\left(x_{0}, \ldots, x_{n}\right)$ there are only $J\left(x_{0}\right)+\cdots+J\left(x_{n}\right)$ non-zero terms in the sum

$$
H\left(x_{0}, \ldots, x_{n}\right):=\sum_{i=0}^{\infty} H\left(f_{i}, f_{i+1}\right)\left(x_{0}, \ldots, x_{n}\right) .
$$

The linear map $H: \tilde{C}_{\bullet}(X) \rightarrow \tilde{C}_{\bullet}(X)$ of degree 1 so defined satisfies

$$
\delta H+H \delta=\sum_{i=0}^{\infty}\left(f_{i+1}\right)_{*}-\left(f_{i}\right)_{*}=\lim _{i \rightarrow \infty}\left(f_{i}\right)_{*}-\left(f_{0}\right)_{*}=\mathrm{id}
$$

by (2). That is, it is a contracting homotopy for $\tilde{C}_{\bullet}(X)$. If $H$ extends to a bounded linear map $H: \mathscr{S}^{k+m} \tilde{C}_{\bullet}(X) \rightarrow \mathscr{S}^{k} \tilde{C} \bullet(X)$, then it is the desired chain homotopy between the zero map and the embedding $\mathscr{S}^{k+m} \tilde{C}_{\bullet}(X) \rightarrow \mathscr{S}^{k} \tilde{C}_{\bullet}(X)$.

Since the maps $f_{i}, f_{i+1}$ are uniformly quasi-Lipschitz and close, the control condition on elements of $\mathscr{S}^{k} \tilde{C}(X)$ is uniformly preserved by all operators $H\left(f_{i}, f_{i+1}\right)$ and hence by $H$. It therefore remains to consider the growth condition. For any $R>0$, the unit ball of $\mathscr{S}^{k+m} C_{n}(X)_{R}$ is the complete absolutely convex hull of the rescaled basis vectors $\left(\ell\left(x_{0}\right)+1\right)^{-k-m} \cdot\left(x_{0}, \ldots, x_{n}\right)$ with $x_{0}, \ldots, x_{n} \in X$ satisfying $d\left(x_{i}, x_{j}\right) \leq R$. Hence $H$ is bounded on $\mathscr{S}^{k+m} C_{n}(X)_{R}$ if (and only if) the images of these rescaled basis vectors form a uniformly bounded subset of $\mathscr{S}^{k} C_{n+1}(X)$.

There is $C>0$ with $\ell\left(f_{i}(x)\right)+1 \leq C(\ell(x)+1)$ for all $i \in \mathbb{N}, x \in X$. This follows from the uniform quasi-Lipschitz condition and the finiteness of the set of $f_{i}(*)$, $i \in \mathbb{N}$. Hence the set of

$$
(-1)^{j}\left(\ell\left(x_{0}\right)+1\right)^{-k} \cdot\left(f_{i}\left(x_{0}\right), \ldots, f_{i}\left(x_{j}\right), f_{i+1}\left(x_{j}\right), \ldots, f_{i+1}\left(x_{n}\right)\right)
$$


for $x_{0}, \ldots, x_{n} \in X$ with $d\left(x_{i}, x_{j}\right) \leq R$ is uniformly bounded in $\mathscr{S}^{k} C_{n}(X)$. The image of $\left(\ell\left(x_{0}\right)+1\right)^{-k} \cdot H\left(x_{0}, \ldots, x_{n}\right)$ is a sum of $J\left(x_{0}\right)+\cdots+J\left(x_{n}\right)$ of these basis vectors. This number is $O\left(\ell\left(x_{0}\right)+1\right)^{m}$ by hypothesis. Hence the set of $\left(\ell\left(x_{0}\right)+1\right)^{-k-m} \cdot H\left(x_{0}, \ldots, x_{n}\right)$ for $x_{0}, \ldots, x_{n} \in X$ with $d\left(x_{i}, x_{j}\right) \leq R$ is uniformly bounded in $\mathscr{S}^{k} C_{n+1}(X)$ as desired.

\section{TEMPERED GROUP COHOMOLOGY AND PROJECTIVE RESOLUTIONS}

Let $G$ be a finitely generated discrete group. Recall the following standard definition of $H^{n}(G)$. Let $\mathscr{C}^{n}(G)$ be the space of all functions $G^{n} \rightarrow \mathbb{R}$, with the convention $\mathscr{C}^{0}(G)=\mathbb{R}$; define $\delta: \mathscr{C}^{n}(G) \rightarrow \mathscr{C}^{n+1}(G)$ by

$$
\begin{aligned}
\delta \phi\left(g_{1}, \ldots, g_{n+1}\right) & :=\phi\left(g_{2}, \ldots, g_{n}\right) \\
& +\sum_{j=1}^{n}(-1)^{j} \phi\left(g_{1}, \ldots, g_{j} \cdot g_{j+1}, \ldots, g_{n+1}\right)+(-1)^{n+1} \phi\left(g_{1}, \ldots, g_{n}\right) .
\end{aligned}
$$

Then $\mathscr{C}^{\bullet}(G):=\left(\mathscr{C}^{n}(G), \delta\right)_{n \geq 0}$ is a chain complex with $H^{n}\left(\mathscr{C}^{\bullet}(G)\right) \cong H^{n}(G)$.

We are interested in subcomplexes of $\mathscr{C} \bullet(G)$ defined by growth conditions. Let $\mathscr{C}^{n}\left(\mathscr{S}^{k} G\right) \subseteq \mathscr{C}^{n}(G)$ be the subspace of cochains $\phi: G^{n} \rightarrow \mathbb{R}$ that satisfy

$$
\left|\phi\left(g_{1}, \ldots, g_{n}\right)\right| \leq C\left(\ell\left(g_{1}\right)+1\right)^{k} \cdots\left(\ell\left(g_{n}\right)+1\right)^{k}
$$

for some $C>0$ and for $k \in \mathbb{R}_{+}$. We let $\mathscr{C}^{n}\left(\mathscr{S}^{\infty} G\right)=\bigcup_{k \in \mathbb{N}} \mathscr{C}^{n}\left(\mathscr{S}^{k} G\right)$. We let $\mathscr{C}^{n}\left(\mathscr{S}^{\omega} G\right)$ be the subspace of $\phi: G^{n} \rightarrow \mathbb{R}$ that satisfy

$$
\left|\phi\left(g_{1}, \ldots, g_{n}\right)\right| \leq C \alpha^{\ell\left(g_{1}\right)} \cdots \alpha^{\ell\left(g_{n}\right)}=C \alpha^{\ell\left(g_{1}\right)+\cdots+\ell\left(g_{n}\right)}
$$

for all $\alpha>1$. We define $\mathscr{C}^{n}(\mathscr{O} G)$ to consist of those $\phi: G^{n} \rightarrow \mathbb{R}$ that satisfy (4) for some $\alpha>1$. One checks immediately that the boundary map $\delta$ preserves these growth conditions, so that we get chain subcomplexes of $\mathscr{C} \bullet(G)$. We let $H^{*}\left(\mathscr{S}^{k} G\right)$ for $k \in \mathbb{R}_{+} \cup\{\infty, \omega\}, * \in \mathbb{N}$, and $H^{*}(\mathscr{O} G)$ for $* \in \mathbb{N}$ be the resulting cohomology groups. The cohomology $H^{*}\left(\mathscr{S}^{0} G\right)=H^{*}\left(\ell_{1} G\right)$ is the bounded cohomology of $G$. We call $H^{*}\left(\mathscr{S}^{\infty} G\right)$ polynomial growth cohomology, $H^{*}\left(\mathscr{S}^{\omega} G\right)$ subexponential growth cohomology, and $H^{*}(\mathscr{O} G)$ exponential growth cohomology. There are natural maps $H^{*}\left(\mathscr{S}^{k} G\right) \rightarrow H^{*}\left(\mathscr{S}^{l} G\right) \rightarrow H^{*}(\mathscr{O} G) \rightarrow H^{*}(G)$ for $k, l \in \mathbb{R}_{+} \cup\{\infty, \omega\}$ with $l \geq k$. We want to find sufficient conditions for some of them to be isomorphisms.

First we rewrite the chain complexes above in terms of bar resolutions. Let $A$ be a bornological unital algebra and let $M$ be a bornological left module over $A$. The bar resolution $\operatorname{Bar}_{\bullet}(A, M)$ of $M$ is defined as follows. Let $\operatorname{Bar}_{n}(A, M):=A^{\hat{\otimes} n+1} \hat{\otimes} M$ for $n \geq 0$ and view this as a left $A$-module by $a \cdot\left(x_{0} \otimes \cdots \otimes x_{n+1}\right):=\left(a \cdot x_{0}\right) \otimes x_{1} \otimes \cdots \otimes x_{n+1}$. Here $\hat{\otimes}$ denotes the projective complete bornological tensor product (see 6 ). We also define $\operatorname{Bar}_{-1}(A, M)=M$. The boundary map $b^{\prime}: \operatorname{Bar}_{n}(A, M) \rightarrow \operatorname{Bar}_{n-1}(A, M)$ is defined by

$$
b^{\prime}\left(x_{0} \otimes \cdots \otimes x_{n+1}\right):=\sum_{j=0}^{n}(-1)^{j} x_{0} \otimes \cdots \otimes x_{j} \cdot x_{j+1} \otimes \cdots \otimes x_{n+1}
$$

for all $n \geq 0, x_{0}, \ldots, x_{n} \in A, x_{n+1} \in M$. Then $\left(b^{\prime}\right)^{2}=0$ and $b^{\prime}$ is a left module homomorphism, so that we have a chain complex of bornological left $A$-modules. The map $s\left(x_{0} \otimes \cdots \otimes x_{n}\right):=1 \otimes x_{0} \otimes \cdots \otimes x_{n}$ is a bounded contracting homotopy, that is, $s b^{\prime}+b^{\prime} s=$ id. Moreover, $\operatorname{Bar}_{n}(A, M)$ is a free module for all $n \geq 0$. Thus the bar resolution is a free bornological left $A$-module resolution of $M$. Let $N$ be another bornological left $A$-module and let $\operatorname{Hom}_{A}\left(\operatorname{Bar}_{\bullet}(A, M), N\right)$ be the chain complex of bounded left module homomorphisms $f: \operatorname{Bar}_{n}(A, M) \rightarrow N$ for $n \in \mathbb{N}$. We let $\operatorname{Ext}_{A}^{n}(M, N)$ be the $n$th cohomology of $\operatorname{Hom}_{A}(\operatorname{Bar} \bullet(A, M), N)$. 
Since

$$
\operatorname{Hom}_{A}(A \hat{\otimes} X, N) \cong \operatorname{Hom}(X, N)
$$

for any bornological vector space $X$ and any bornological left $A$-module $N$, free modules are projective for extensions with a bounded linear section. The same proof as in Abelian categories shows that any two free resolutions of the same module are homotopy equivalent via bounded $A$-linear chain maps and homotopies. Therefore, if $P_{\bullet} \rightarrow M$ is any free resolution of $M$ (this means that we have a bounded contracting homotopy), then the chain complex $\operatorname{Hom}_{A}\left(P_{\bullet}, N\right)$ is naturally homotopy equivalent to $\operatorname{Hom}_{A}(\operatorname{Bar} \bullet(A, M), N)$, so that $\operatorname{Ext}_{A}^{n}(M, N)$ is naturally isomorphic to the cohomology of the chain complex $\operatorname{Hom}_{A}\left(P_{\bullet}, N\right)$.

Lemma 6. Equip $\mathbb{R}$ with the trivial representation of $G$. Then

$$
H^{n}(G) \cong \operatorname{Ext}_{\mathbb{R}[G]}^{n}(\mathbb{R}, \mathbb{R}), \quad H^{n}(\mathscr{T} G) \cong \operatorname{Ext}_{\mathscr{T}(G)}^{n}(\mathbb{R}, \mathbb{R})
$$

for $\mathscr{T}(G)=\mathscr{O}(G)$ or $\mathscr{T}(G)=\mathscr{S}^{k}(G), k \in \mathbb{R}_{+} \cup\{\infty, \omega\}$.

Proof. We write $H^{n}(\mathbb{R}[G])=H^{n}(G)$ and allow $\mathscr{T}(G)=\mathbb{R}[G]$ among our list of convolution algebras. We claim that the chain complex $\operatorname{Hom}_{\mathscr{T}(G)}\left(\operatorname{Bar}_{\bullet}(\mathscr{T}(G), \mathbb{R}), \mathbb{R}\right)$ is canonically isomorphic to the chain complexes that we have used above to define $H^{n}(\mathscr{T} G)$. This establishes the lemma.

Consider $\mathbb{R}[G]$ first. Then $\operatorname{Bar}_{n}(\mathbb{R}[G], \mathbb{R})=\mathbb{R}[G]^{\hat{\otimes} n+1}$. This is isomorphic to $\mathbb{R}\left[G^{n+1}\right]$ with the fine bornology. A $G$-equivariant linear map to $\mathbb{R}$ is the same as a $G$-invariant linear functional. Such linear functionals on $\mathbb{R}\left[G^{n+1}\right]$ are of the form

$$
\psi \mapsto \sum_{g_{0}, \ldots, g_{n} \in G} \psi\left(g_{0}, \ldots, g_{n}\right) \phi\left(g_{1}, \ldots, g_{n}\right)
$$

for a uniquely determined function $\phi: G^{n} \rightarrow \mathbb{R}$. Thus $\operatorname{Hom}_{\mathbb{R}[G]}\left(\operatorname{Bar}_{n}(\mathbb{R}[G], \mathbb{R}), \mathbb{R}\right) \cong$ $\mathscr{C}^{n}(G)$. One checks immediately that these isomorphisms form a chain map.

For another choice of $\mathscr{T}(G)$, the complex $\operatorname{Bar}_{\bullet}(\mathscr{T}(G), \mathbb{R})$ contains $\operatorname{Bar} \bullet(\mathbb{R}[G], \mathbb{R})$ as a dense subcomplex. Hence we may view $\operatorname{Hom}_{\mathscr{T}(G)}\left(\operatorname{Bar}_{\bullet}(\mathscr{T}(G), \mathbb{R}), \mathbb{R}\right)$ as a subcomplex of $\operatorname{Hom}_{\mathbb{R}[G]}\left(\operatorname{Bar}_{\bullet}(\mathbb{R}[G], \mathbb{R}), \mathbb{R}\right) \cong \mathscr{C} \bullet(G)$. We claim that it agrees with the subcomplex that defines $H^{n}(\mathscr{T} G)$. The space $\operatorname{Hom}_{\mathscr{T}(G)}\left(\operatorname{Bar}_{n}(\mathscr{T}(G), \mathbb{R}), \mathbb{R}\right)$ is the space of all $\phi: G^{n} \rightarrow \mathbb{R}$ for which (5) defines a bounded linear functional on $\mathscr{T}(G)^{\hat{\otimes} n}$. Equivalently, the $n$-linear map

$$
\left(\psi_{1}, \ldots, \psi_{n}\right) \mapsto \sum_{g_{1}, \ldots, g_{n} \in G} \psi_{1}\left(g_{1}\right) \cdots \psi_{n}\left(g_{n}\right) \phi\left(g_{1}, \ldots, g_{n}\right)
$$

is bounded on $\mathscr{T}(G)^{n}=\mathscr{T}(G) \times \cdots \times \mathscr{T}(G)$. We have identified the dual space of $\mathscr{T}(G)$ in Section 2. The same discussion applies to multilinear maps and yields the assertions of the lemma.

Theorem 7. Let $G$ be a finitely generated discrete group. If $\mathscr{T} \tilde{C} \bullet(G)$ has a bounded contracting homotopy, then the canonical map $H^{n}(\mathscr{T} G) \rightarrow H^{n}(G)$ is an isomorphism.

Proof. Let $G$ act diagonally on $\mathscr{T} C_{n}(G)$ by $g \cdot\left(x_{0}, \ldots, x_{n}\right):=\left(g x_{0}, \ldots, g x_{n}\right)$. Let $C_{n}^{\prime}(G) \subseteq C_{n}(G)$ be the subspace spanned by $\left(1, x_{1}, \ldots, x_{n}\right)$. Let $C_{n}^{\prime}(G)_{R}$ for $R>0$ be the finite-dimensional subspace spanned by $\left(1, x_{1}, \ldots, x_{n}\right)$ with $d\left(x_{i}, x_{j}\right) \leq R$ and $d\left(1, x_{i}\right) \leq R$ for all $i, j \in\{1, \ldots, n\}$. There is a bornological isomorphism

$$
\mathscr{T}(G) \hat{\otimes} C_{n}^{\prime}(G)_{R} \rightarrow \mathscr{T} C_{n}(G)_{R}, \quad g \otimes\left(1, x_{1}, \ldots, x_{n}\right) \mapsto\left(g, g x_{1}, \ldots, g x_{n}\right)
$$

because of the left invariance of the metric on $G$. This implies

$$
\mathscr{T} C_{n}(G) \cong \lim _{\longrightarrow} \mathscr{T}(G) \hat{\otimes} C_{n}^{\prime}(G)_{R} \cong \mathscr{T}(G) \hat{\otimes} C_{n}^{\prime}(G)
$$


because $\hat{\otimes}$ commutes with direct limits. If we give $\mathscr{T}(G) \hat{\otimes} C_{n}^{\prime}(G)$ the standard $\mathscr{T}(G)$-module structure via $\phi \cdot\left(\phi^{\prime} \otimes \psi\right):=\left(\phi * \phi^{\prime}\right) \otimes \psi$, then the above isomorphism is a module homomorphism. Hence $\mathscr{T} C_{n}(G)$ is a free $\mathscr{T}(G)$-module. It is easy to see that the boundary maps of $\mathscr{T} C_{\bullet}(G)$ and the augmentation map $\mathscr{T} C_{0}(G) \rightarrow \mathbb{R}$ are $G$-equivariant and hence $\mathscr{T}(G)$-module homomorphisms.

Since $\tilde{C}_{\bullet}(G)$ is contractible, $C_{\bullet}(G) \rightarrow \mathbb{R}$ is a free $\mathbb{R}[G]$-module resolution of $\mathbb{R}$. (It is isomorphic to the reduced bar resolution.) Since all free resolutions are equivalent, Lemma 6 yields $H^{n}(G) \cong H^{n}\left(\operatorname{Hom}_{\mathbb{R}[G]}(C \bullet(G), \mathbb{R})\right)$. The canonical isomorphisms

$$
\operatorname{Hom}_{\mathbb{R}[G]}\left(\mathbb{R}[G] \hat{\otimes} C_{n}^{\prime}(G), \mathbb{R}\right) \cong \operatorname{Hom}\left(C_{n}^{\prime}(G), \mathbb{R}\right) \cong \operatorname{Hom}_{\mathscr{T}(G)}\left(\mathscr{T}(G) \hat{\otimes} C_{n}^{\prime}(G), \mathbb{R}\right)
$$

are compatible with the boundary maps on $C_{\bullet}(G)$ and $\mathscr{T} C_{\bullet}(G)$, that is, they form an isomorphism of chain complexes

$$
\operatorname{Hom}_{\mathbb{R}[G]}(C \bullet(G), \mathbb{R}) \cong \operatorname{Hom}_{\mathscr{T}(G)}(\mathscr{T} C \bullet(G), \mathbb{R}) .
$$

If $\mathscr{T} \tilde{C}_{\bullet}(G)$ has a bounded contracting homotopy, then $\mathscr{T} C \bullet(G)$ augmented by $\alpha: \mathscr{T} C_{0} \rightarrow \mathbb{R}$ is a free $\mathscr{T}(G)$-module resolution of $\mathbb{R}$. By the uniqueness of free resolutions, this implies $H^{n}(\mathscr{T} G) \cong H^{n}\left(\operatorname{Hom}_{\mathscr{T}(G)}\left(\mathscr{T} C_{\bullet}(G), \mathbb{R}\right)\right)$. The assertion of the theorem now follows from 6 .

Corollary 8. If $G$ has a combing of polynomial growth, then $H^{n}\left(\mathscr{S}^{\infty}(G)\right) \cong H^{n}(G)$. If $G$ has a combing of subexponential growth, then $H^{n}\left(\mathscr{S}^{\omega}(G)\right) \cong H^{n}(G)$. If $G$ has a combing of exponential growth, then $H^{n}(\mathscr{O}(G)) \cong H^{n}(G)$.

Proof. Combine Theorems 5 and 7.

The following theorem provides an obstruction for the contractibility of $\mathscr{T} \tilde{C}_{\bullet}(G)$. Actually, the proof shows that it even yields an obstruction to $H^{n}(\mathscr{T} G) \rightarrow H^{n}(G)$ being a bornological isomorphism.

Theorem 9. Let $G$ be a finitely generated discrete group and let $\mathscr{T}(G)$ be one of $\mathscr{S}^{\infty}(G)$, $\mathscr{S}^{\omega}(G)$, or $\mathscr{O}(G)$. Suppose that $\mathscr{T} \tilde{C} \bullet(G)$ is contractible. Then $H^{n}(G)$ is finite-dimensional for all $n \in \mathbb{N}$.

Proof. By Theorem 7, $H^{n}(G)$ and $H^{n}(\mathscr{T} G)$ are isomorphic. Even more, they are computed by chain homotopy equivalent bornological chain complexes. The cohomology of a bornological chain complex carries a canonical bornology, which may fail to be separated. The homotopy equivalence of the underlying bornological chain complexes implies that $H^{n}(G)$ and $H^{n}(\mathscr{T} G)$ are isomorphic as bornological vector spaces. Replacing $\mathscr{T}(G)$ by $\mathscr{O}(G)$, we no longer necessarily have an isomorphism, but we obtain that $H^{n}(G)$ is a direct summand in $H^{n}(\mathscr{O} G)$ because $\mathbb{R}[G] \subseteq \mathscr{O}(G) \subseteq$ $\mathscr{T}(G)$.

The group cohomology $H^{n}(G)$ is the dual space of the group homology of $G$. The latter is isomorphic to $\mathbb{R}[B]$ for some countable set $B$. The dual space of $\mathbb{R}[B]$ is $\prod_{x \in B} \mathbb{R}$, equipped with the product bornology. We get a bornological isomorphism $H^{n}(G) \cong \prod_{x \in B} \mathbb{R}$. Thus $H^{n}(G)$ is a Fréchet-Schwartz space. In contrast, the complex that computes $H^{n}(\mathscr{O} G)$ is a complex of Silva spaces. Hence any separated subspace of $H^{n}(\mathscr{O} G)$ is a Silva space. In particular, $H^{n}(G)$ is a Fréchet-Schwartz and a Silva space at the same time. We claim that such a space is necessarily finite-dimensional.

Let $V$ be a Fréchet-Schwartz space whose von Neumann bornology is a Silva bornology. Thus $V=\lim _{n} V_{n}$ for an inductive system $\left(V_{n}\right)_{n \in \mathbb{N}}$ of Banach subspaces with injective maps $\overrightarrow{V_{n}} \rightarrow V_{n+1}$. Let $S_{n}$ be the unit ball of $V_{n}$. Since $V$ is a Fréchet space, there exists a sequence of positive scalars $\left(\epsilon_{n}\right)$ such that $\bigcup \epsilon_{n} S_{n}$ is still von Neumann bounded. This is the bornological definition of metrisability, see [9]. However, any bounded subset of $\lim _{\longrightarrow} V_{n}$ is absorbed by $S_{N}$ for some $N \in \mathbb{N}$. 
Hence $S_{N}$ absorbs $S_{n}$ for all $n \in \mathbb{N}$. Thus $V$ is isomorphic to the Banach space $V_{N}$. Since $V$ is Fréchet-Schwartz, any bounded subset in $V$ is relatively compact. However, the only Banach spaces with relatively compact unit ball are the finite-dimensional ones. Hence $V$ is finite-dimensional as asserted.

It is known that $B G$ has finite type if $G$ is combable. That is, $B G$ has the homotopy type of a simplicial complex with finite skeleta. Of course, this implies that $H^{n}(G) \cong H^{n}(B G)$ is finite-dimensional. Other popular conditions of geometric group theory like amenability or exactness do not guarantee $B G$ to have finite-dimensional cohomology spaces and hence cannot possibly suffice to prove contractibility of $\mathscr{T} \tilde{C}_{\bullet}(G)$. Only conditions that guarantee some finiteness of $B G$ have a chance to imply contractibility of $\mathscr{T} \tilde{C} \bullet(G)$.

\section{USING OTHER FREE RESOLUTIONS AND THE EXAMPLE OF FREE GROUPS}

Let $G$ be a finitely generated discrete group and let $\mathscr{T}(G)$ be one of the algebras $\mathscr{O}(G)$ or $\mathscr{S}^{k}(G)$ for $k \in \mathbb{R}_{+} \cup\{\infty, \omega\}$. We want to find simpler chain complexes that are homotopy equivalent to $\mathscr{T} C \bullet(G)$.

Recall that $\operatorname{Mod}(A)$ denotes the category of bornological left $A$-modules. The embedding $i: \mathbb{R}[G] \rightarrow \mathscr{T}(G)$ induces a "forgetful" functor

$$
i^{*}: \operatorname{Mod}(\mathscr{T}(G)) \rightarrow \operatorname{Mod}(\mathbb{R}[G]) .
$$

We claim that $i^{*}$ has a left adjoint functor $i_{!}: \operatorname{Mod}(\mathbb{R}[G]) \rightarrow \operatorname{Mod}(\mathscr{T}(G))$. That is, the functors $i^{*}$ and $i_{\text {! }}$ are related by natural isomorphisms

$$
\operatorname{Hom}_{\mathscr{T}(G)}\left(i_{!}(M), N\right) \cong \operatorname{Hom}_{\mathbb{R}[G]}\left(M, i^{*}(N)\right)
$$

if $M$ and $N$ are bornological modules over $\mathbb{R}[G]$ and $\mathscr{T}(G)$, respectively. We construct $i_{\text {! }}$ as follows (see also $[10]$ ). Let $M$ be a left bornological $\mathbb{R}[G]$-module. We let $\mathscr{T}(G) \hat{\otimes}_{G} M$ be the quotient of $\mathscr{T}(G) \hat{\otimes} M$ by the closed linear subspace generated by $\phi * \delta_{g} \otimes m-\phi \otimes g m$ for $\phi \in \mathscr{T}(G), g \in G, m \in M$. Since we take closures, this quotient is again a complete convex bornological vector space. We turn $\mathscr{T}(G) \hat{\otimes}_{G} M$ into a left bornological $\mathscr{T}(G)$-module by $\phi_{1} \cdot\left(\phi_{2} \otimes m\right):=\phi_{1} * \phi_{2} \otimes m$ for $\phi_{1}, \phi_{2} \in \mathscr{T}(G), m \in M$. We claim that $i_{!}(M)=\mathscr{T}(G) \hat{\otimes}_{G} M$.

To prove this, we verify (7). Let $N$ be a bornological $\mathscr{T}(G)$-module. By the universal property of $\hat{\otimes}$, bounded $\mathscr{T}(G)$-module homomorphisms $\mathscr{T}(G) \hat{\otimes}_{G} M \rightarrow N$ correspond to bilinear maps $f: \mathscr{T}(G) \times M \rightarrow N$ that satisfy

$$
f\left(\phi_{1} * \phi_{2}, m\right)=\phi_{1} \cdot f\left(\phi_{2}, m\right), \quad f\left(\phi * \delta_{g}, m\right)=f(\phi, g \cdot m)
$$

for all $\phi, \phi_{1}, \phi_{2} \in \mathscr{T}(G), g \in G, m \in M$. The first relation implies that $f(\phi, m)=$ $\phi \cdot f\left(\delta_{1}, m\right)$, the second relation implies that the bounded linear map $M \rightarrow N$, $m \mapsto f\left(\delta_{1}, m\right)$, is $G$-equivariant. Conversely, given any bounded, $G$-equivariant linear map $f^{\prime}: M \rightarrow N$, the formula $f(\phi, m):=\phi \cdot f^{\prime}(m)$ defines a bounded $\mathscr{T}(G)$ module homomorphism $\mathscr{T}(G) \hat{\otimes}_{G} M \rightarrow N$. Thus we have constructed the natural isomorphism (7). We usually write $\mathscr{T}(G) \hat{\otimes}_{G} M$ and not $i_{!}(M)$ because we want to keep $\mathscr{T}(G)$ in our notation.

Proposition 10. There is a canonical isomorphism of bornological chain complexes

$$
\mathscr{T}(G) \hat{\otimes}_{G} C_{\bullet}(G) \cong \mathscr{T} C_{\bullet}(G) .
$$

If $P_{\bullet} \rightarrow \mathbb{R}$ is any free $\mathbb{R}[G]$-module resolution of $G$, then $\mathscr{T}(G) \hat{\otimes}_{G} P_{\bullet}$ is bornologically homotopy equivalent to $\mathscr{T} C_{\bullet}(G)$ (the chain maps and chain homotopies involved are bounded linear maps).

Proof. During the proof of Theorem 7, we have shown

$$
C_{n}(G) \cong \mathbb{R}[G] \hat{\otimes} C_{n}^{\prime}(G), \quad \mathscr{T} C_{n}(G) \cong \mathscr{T}(G) \hat{\otimes} C_{n}^{\prime}(G),
$$


for certain fine bornological vector spaces $C_{n}^{\prime}(G)$; these are module isomorphisms with respect to the free module structures on the right hand sides. It follows easily that $\mathscr{T} C_{n}(G) \cong \mathscr{T}(G) \hat{\otimes}_{G} C_{n}(G)$. These isomorphisms form an isomorphism of chain complexes $\mathscr{T} C_{\bullet}(G) \cong \mathscr{T}(G) \hat{\otimes}_{G} C_{\bullet}(G)$. This implies the second assertion because of the uniqueness up to homotopy equivalence of free resolutions.

Example 11. Let $r \in \mathbb{N}_{\geq 1}$ and let $\mathbb{F}_{r}$ be the non-Abelian free group on $r$ generators, denoted $s(1), \ldots, s(r)$. Let $\mathscr{T}\left(\mathbb{F}_{r}\right)$ be one of the convolution algebras considered above. Example 4 provides a combing of linear growth on $\mathbb{F}_{r}$. Theorem 5 yields that $\mathscr{T} \tilde{C} \bullet\left(\mathbb{F}_{r}\right)$ is contractible if $\mathscr{T}\left(\mathbb{F}_{r}\right)$ is $\mathscr{S}^{\infty}\left(\mathbb{F}_{r}\right), \mathscr{S}^{\omega}\left(\mathbb{F}_{r}\right)$, or $\mathscr{O}\left(\mathbb{F}_{r}\right)$. We are going to show that this fails for $\mathscr{S}^{k}\left(\mathbb{F}_{r}\right)$ with $k \in \mathbb{R}_{+}$and, especially, for $\ell_{1}\left(\mathbb{F}_{r}\right)$.

The Cayley graph of $\mathbb{F}_{r}$ with respect to the generators above is a tree. The simplicial chain complex associated to this tree is a free $\mathbb{R}\left[\mathbb{F}_{r}\right]$-module resolution of the trivial representation. Explicitly, this yields the free resolution

$$
\cdots \longrightarrow 0 \longrightarrow \mathbb{R}\left[\mathbb{F}_{r}\right]^{r} \stackrel{b_{1}}{\longrightarrow} \mathbb{R}\left[\mathbb{F}_{r}\right] \stackrel{b_{0}}{\longrightarrow} \mathbb{R} \longrightarrow 0,
$$

where

$$
b_{0}(\phi)=\sum_{g \in \mathbb{F}_{r}} \phi(g), \quad b_{1}\left(\phi_{1}, \ldots, \phi_{r}\right)(g)=\sum_{j=1}^{r} \phi_{j}(g)-\phi_{j}(g s(j)) .
$$

It is clear that the maps $b_{0}$ and $b_{1}$ are module homomorphisms and that $b_{0} b_{1}=0$. To check by hand that $(8)$ is a resolution, we specify a linear map $\sigma: \mathbb{R}\left[\mathbb{F}_{r}\right] \rightarrow \mathbb{R}\left[\mathbb{F}_{r}\right]^{r}$ that satisfies $\sigma b_{1}=\mathrm{id}$ and $b_{1} \sigma(g)=g-1$ for all $g \in \mathbb{F}_{r}$. Actually, such a map is necessarily unique. We can obtain $\sigma(g)$ for $g \in \mathbb{F}_{r}$ by induction on the length of $g$. If $g=1$, then $\sigma(g)=0$. Otherwise, write $g=g_{2} s(j)^{\epsilon}$ with $j \in\{1, \ldots, r\}, \epsilon \in\{ \pm 1\}$, and $\ell\left(g_{2}\right)<\ell(g)$. Then we let $\sigma(g)-\sigma\left(g_{2}\right)$ be equal to $g$ in the $j$ th summand if $\epsilon=1$, and $-g_{2}$ in the $j$ th summand if $\epsilon=-1$. This determines $\sigma(g)$ for all $g \in \mathbb{F}_{r}$. The relation $\sigma b_{1}=$ id is trivial, and $b_{1} \sigma(g)=g-1$ follows by induction on $\ell(g)$.

Proposition 10 yields that $\mathscr{T} C \bullet \bullet\left(\mathbb{F}_{r}\right)$ is chain homotopy equivalent to

$$
\cdots \longrightarrow 0 \longrightarrow \mathscr{T}\left(\mathbb{F}_{r}\right)^{r} \stackrel{b_{1}}{\longrightarrow} \mathscr{T}\left(\mathbb{F}_{r}\right) \text {. }
$$

A chain complex equivalent to $\mathscr{T} \tilde{C}_{\bullet}\left(\mathbb{F}_{r}\right)$ is obtained by replacing $\mathscr{T}\left(\mathbb{F}_{r}\right)$ by the kernel of the augmentation map $b_{0}: \mathscr{T}\left(\mathbb{F}_{r}\right) \rightarrow \mathbb{R}$. As a result, $\mathscr{T} \tilde{C} \bullet\left(\mathbb{F}_{r}\right)$ is split exact except possibly in dimensions 0 and 1 . It is split exact in all dimensions if and only if $b_{1}$ is a bornological isomorphism from $\mathscr{T}\left(\mathbb{F}_{r}\right)^{r}$ onto ker $b_{0} \subseteq \mathscr{T}\left(\mathbb{F}_{r}\right)$.

The construction above shows that $\sigma(g)$ is a sum of exactly $\ell(g)$ different standard basis vectors of $\mathbb{R}\left[\mathbb{F}_{r}\right]^{r}$. Hence $\sigma$ is a bounded linear map for $\mathscr{O}\left(\mathbb{F}_{r}\right), \mathscr{S}^{\infty}\left(\mathbb{F}_{r}\right)$, and $\mathscr{S}^{\omega}\left(\mathbb{F}_{r}\right)$, and maps $\mathscr{S}^{k+1}\left(\mathbb{F}_{r}\right) \rightarrow \mathscr{S}^{k}\left(\mathbb{F}_{r}\right)^{r}$ for $k \in \mathbb{R}_{+}$. These assertions are already contained in Theorem 5 . The map $\sigma$ is, in fact, closely related to the combing for $\mathbb{F}_{r}$ constructed in Example 4

Let $\mathscr{T}\left(\mathbb{F}_{r}\right)=\mathscr{S}^{k}\left(\mathbb{F}_{r}\right)$ for $k \in \mathbb{R}_{+}$. Since $\sigma$ is a bounded linear map from $\mathscr{S}^{k}\left(\mathbb{F}_{r}\right)$ to $\mathscr{S}^{k-1}\left(\mathbb{F}_{r}\right)^{r}$, the map $b_{1}$ is injective on $\mathscr{S}^{k}\left(\mathbb{F}_{r}\right)^{r}$. Therefore, the chain complex $\mathscr{T} C_{\bullet}\left(\mathbb{F}_{r}\right)$ is split exact in degree 1 in the sense that the boundary map $\mathscr{S}^{k} C_{2}\left(\mathbb{F}_{r}\right) \rightarrow \operatorname{ker} \delta \subseteq \mathscr{S}^{k} C_{1}\left(\mathbb{F}_{r}\right)$ is split surjective. Since 8$)$ is exact, $b_{1}\left(\mathscr{S}^{k}\left(\mathbb{F}_{r}\right)^{r}\right)$ contains $b_{1}\left(\mathbb{R}\left[\mathbb{F}_{r}\right]^{r}\right)=\operatorname{ker} b_{0} \cap \mathbb{R}\left[\mathbb{F}_{r}\right]$, which is dense in ker $b_{0}: \mathscr{S}^{k}\left(\mathbb{F}_{r}\right) \rightarrow \mathbb{R}$. We have $b_{1}\left(\mathscr{S}^{k}\left(\mathbb{F}_{r}\right)^{r}\right) \neq \operatorname{ker} b_{0}$ already for $r=1$, that is, for the group of integers; this amounts to a well-known fact about Fourier series. Thus $H_{0}\left(\mathscr{S}^{k} \tilde{C}_{\bullet}\left(\mathbb{F}_{r}\right)\right)$ is non-trivial, but its separated quotient (called reduced homology in [13]) vanishes.

\section{Tempered CEllular CHAin COMPleXes}

Proposition 10 allows us to replace $\mathscr{T} C \bullet(G)$ by simpler chain complexes for a discrete group $G$. For more general metric spaces, we do not have the category of $\mathbb{R}[G]$-modules and hence cannot speak of projective resolutions. Therefore, we 
have to work harder to find simpler models for $\mathscr{T} C \bullet(X)$. In this section, we relate $\mathscr{T} C \bullet(X)$ to tempered variants of cellular chain complexes. The motivation for this is two-fold. These complexes sometimes arise in applications, and we may want to use Theorem 5 to get information about them. Conversely, if $X$ is not known to be combable, we may want to check the contractibility of $\mathscr{T} \tilde{C} \bullet \bullet(X)$ for a smaller complex that is homotopy equivalent to it.

As a preparation, we briefly recall the construction of cellular chain complexes. Let $X$ be a CW-complex. We denote the set of closed $n$-cells in $X$ by $X_{n}$ and write $X^{(n)}$ for the $n$th skeleton of $X$. Thus $X^{(0)} \subseteq X$ is a discrete subset. A continuous map $f: X \rightarrow Y$ is called cellular if it maps $X^{(n)}$ to $Y^{(n)}$ for all $n \in \mathbb{N}$. Cellular homotopies are defined using a canonical cell decomposition of $X \times[0,1]$. It is well-known that any continuous map between CW-complexes is homotopic to a cellular map and that homotopic cellular maps are homotopic by a cellular homotopy.

Let $f: X \rightarrow Y$ be cellular. For cells $D \in X_{n}, E \in Y_{n}$, one defines the incidence number $\iota(f, D, E)$ using the action of $f$ on the singular homology of the CW-pairs $\left(X^{(n)}, X^{(n-1)}\right)$ and $\left(Y^{(n)}, Y^{(n-1)}\right)$. Let $\iota(\partial D, F)$ for $D \in X_{n+1}, F \in X_{n}$ be the incidence number of the characteristic map $S^{n} \rightarrow \partial D \subseteq X$. The cellular chain complex $\left(\operatorname{Cell}_{\bullet}(X), \delta\right)_{n \geq 0}$ of $X$ is defined by $\operatorname{Cell}_{n}(X)=\mathbb{R}\left[X_{n}\right]$ and

$$
\delta: \operatorname{Cell}_{n+1}(X) \rightarrow \operatorname{Cell}_{n}(X), \quad \delta(D):=\sum_{F \in X_{n}} \iota(\partial D, F) F .
$$

A cellular map $f: X \rightarrow Y$ induces a chain map

$$
f_{*}: \operatorname{Cell}_{\bullet}(X) \rightarrow \operatorname{Cell}_{\bullet}(Y), \quad f_{*}(D):=\sum_{E \in Y_{n}} \iota(f, D, E) E .
$$

This construction is functorial, that is, $\mathrm{id}_{*}=\mathrm{id}$ and $(f g)_{*}=f_{*} g_{*}$. A cellular homotopy $F: X \times[0,1] \rightarrow Y$ induces a chain homotopy $H(F): \operatorname{Cell}_{\bullet}(X) \rightarrow$ Cell. $_{\bullet}(Y)$ between $\left(F_{0}\right)_{*}$ and $\left(F_{1}\right)_{*}$, that is, $\delta H(F)+H(F) \delta=\left(F_{1}\right)_{*}-\left(F_{0}\right)_{*}$.

For a cellular map $f: X \rightarrow Y$ and $n \in \mathbb{N}$, we let

$$
\|f\|_{n}:=\sup _{D \in X_{n}} \sum_{E \in Y_{n}}|\iota(f, D, E)|
$$

We define similar norms for cellular homotopies and the boundary map $\delta$. We call $f, F$, or $\delta$ bounded if all these norms remain finite. For infinite $\mathrm{CW}$-complexes, this depends on the chosen cell decomposition: if $\left(D_{j}\right)$ is a sequence of different $n$-cells and if we subdivide $D_{j}$ into $j$ cells for each $j$, then the identity map becomes an unbounded cellular map. Boundedness of $\delta$ is automatic for simplicial and cubical cell complexes because the boundaries of an $n$-simplex or an $n$-cube have at most $n+1$ or $2^{n}$ nondegenerate faces, respectively.

Recall that the chain complex $C_{\bullet}(X)$ for a set $X$ is the reduced simplicial chain complex of a certain simplicial set $S(X)$. If we view the simplicial realisation $|S(X)|$ as a CW-complex, then we get $C_{\bullet}(X)=$ Cell $\bullet(|S(X)|)$. We now describe some additional structure on $|S(X)|$ that allows us to define the tempered chain complexes $\mathscr{T} C \bullet(X)$. First, we use the filtration $|S(X)|=\bigcup\left|S(X)_{R}\right|$, where $S(X)_{R}$ is the simplicial subset whose nondegenerate $n$-simplices are only those $\left(x_{0}, \ldots, x_{n}\right)$ with $n \leq R$ and $d\left(x_{i}, x_{j}\right) \leq R$ for all $i, j \in\{0, \ldots, n\}$. The subcomplexes $\left|S(X)_{R}\right|$ are locally finite because bounded subsets of $X$ are finite. Up to quasi-isometry, there is a unique metric on $\left|S(X)_{R}\right|$ for which the cells are uniformly bounded and the embedding $X \rightarrow\left|S(X)_{R}\right|$ is a quasi-isometry. This additional structure on $|S(X)|$ is formalised in the following definition:

Definition 12. A filtered metric $C W$-complex is a CW-complex $X$ together with a filtration $X=\bigcup_{R \in \mathbb{N}} \mathscr{F}_{R} X$ and proper metrics $d_{R}$ on $\mathscr{F}_{R} X$ that define the topology 
of $\mathscr{F}_{R} X$ for all $R \in \mathbb{N}$, such that each $\mathscr{F}_{R} X$ is a locally finite, finite-dimensional subcomplex of $X$ with uniformly bounded cells and bounded boundary map $\delta$ and such that the embeddings $\mathscr{F}_{R} X \rightarrow \mathscr{F}_{R+1} X$ are quasi-isometries for all $R \in \mathbb{N}$.

A morphism $f: X \rightarrow Y$ between filtered metric CW-complexes is a cellular map with the property that for any $R \in \mathbb{N}$ there exists $R^{\prime} \in \mathbb{N}$ such that $f\left(\mathscr{F}_{R} X\right) \subseteq \mathscr{F}_{R^{\prime}} Y$ and $\left.f\right|_{\mathscr{F}_{R} X}: \mathscr{F}_{R} X \rightarrow \mathscr{F}_{R^{\prime}} Y$ is quasi-Lipschitz and bounded with respect to the norms defined in 10 .

If $X$ is a filtered metric CW-complex, then we turn $X \times[0,1]$ into a filtered metric CW-complex using the standard cell decomposition, the filtration $\left(\mathscr{F}_{R} X\right) \times[0,1]$, and the metrics $d_{R}((x, s),(y, t)):=d_{R}(x, y)+|t-s|$. A homotopy between two morphisms $X \rightarrow Y$ is a morphism $X \times[0,1] \rightarrow Y$.

Up to isomorphism of filtered metric CW-complexes, only the equivalence class of the filtration $\left(\mathscr{F}_{R} X\right)$ and the quasi-isometry classes of the metrics $d_{R}$ matter. Thus the filtered metric CW-complex structure on $|S(X)|$ discussed above is unique up to isomorphism.

Example 13. Let $G$ be a finitely generated discrete group and let $X$ be a CWcomplex equipped with a proper cellular action of $G$ and with only countably many cells. Then $X$ becomes a filtered metric CW-complex in a canonical way.

A $G$-invariant subcomplex of $X$ is $G$-compact if and only if the action of $G$ on its cells has only finitely many orbits. Any finite subcomplex of $X$ is contained in a $G$-invariant $G$-compact subcomplex. Since $X$ has countably many cells, there exists an increasing filtration $X=\bigcup_{R \in \mathbb{N}} \mathscr{F}_{R} X$ where the $\mathscr{F}_{R} X$ are $G$-invariant, $G$-compact subcomplexes. This filtration is unique up to equivalence. It follows from $G$-compactness and properness that the subcomplexes $\mathscr{F}_{R} X$ are locally finite and finite-dimensional and that the boundary map of $\mathscr{F}_{R} X$ is bounded.

Since the action of $G$ on $\mathscr{F}_{R} X$ is proper and $G$-compact, we can find a $G$-invariant metric on $\mathscr{F}_{R} X$ that defines the topology of $\mathscr{F}_{R} X$, such that the maps $G \rightarrow \mathscr{F}_{R} X$, $g \mapsto g x$, are quasi-isometric for all $x \in \mathscr{F}_{R} X$. Any two such metrics are quasiisometric. Since the maps $G \rightarrow \mathscr{F}_{R} X, g \mapsto g x$, for some $x \in \mathscr{F}_{0} X$ are quasiisometric for all $R \in \mathbb{N}$, it follows that the embeddings $\mathscr{F}_{R} X \rightarrow \mathscr{F}_{R+1} X$ are also quasi-isometric.

The above construction is natural, that is, $G$-equivariant cellular maps become morphisms of filtered metric CW-complexes. The same holds for $G$-equivariant cellular homotopies.

Let $X$ be a filtered metric CW-complex and recall that $X_{n}$ denotes its set of $n$-cells. It inherits a filtration $X_{n}=\bigcup \mathscr{F}_{R} X_{n}$. Choose a point $\xi_{D} \in D$ for each $D \in X_{n}$ and view this as a map $X_{n} \rightarrow X, D \mapsto \xi_{D}$. This induces proper discrete metrics on the sets $\mathscr{F}_{R} X_{n}$ for all $R, n \in \mathbb{N}$. Our hypotheses on $X$ ensure that the quasi-isometry classes of these metrics do not depend on the choice of the points $\xi_{D}$. Let

$$
\mathscr{T} \operatorname{Cell}_{n}(X):=\lim _{R \rightarrow \infty} \mathscr{T}\left(\mathscr{F}_{R} X_{n}\right)
$$

where $\mathscr{T}$ is $\mathscr{O}$ or $\mathscr{S}^{k}$ for $k \in \mathbb{R}_{+} \cup\{\infty, \omega\}$.

Lemma 14. Let $X$ be a filtered metric $C W$-complex. Then the space $\mathscr{T C}^{-} \operatorname{ll}_{n}(X)$ is independent of the auxiliary choices and the cellular boundary map extends to a bounded linear map $\delta: \mathscr{T C} \operatorname{Cll}_{n+1}(X) \rightarrow \mathscr{T} \operatorname{Cell}_{n}(X)$ for all $n \in \mathbb{N}$. Thus we obtain a bornological chain complex $\mathscr{T}$ Cell $\bullet(X)=\left(\mathscr{T C e l l}_{n}(X), \delta\right)_{n \geq 0} . A$ morphism $f: X \rightarrow Y$ induces a bounded chain map $f_{*}: \mathscr{T}$ Cell• $(X) \rightarrow \mathscr{T}_{\text {Cell }}(Y)$.

A homotopy $F: X \times[0,1] \rightarrow Y$ between two morphisms induces a bounded chain homotopy between the induced maps $\mathscr{T}$ Cell• $(X) \rightarrow \mathscr{T}$ Cell• $(Y)$. 
Proof. The space $\mathscr{T}\left(\mathscr{F}_{R} X_{n}\right)$ does not depend on the choice of the points $\xi_{D}$ because $\mathscr{T}\left(\mathscr{F}_{R} X_{n}\right)$ only depends on the quasi-isometry class of the metric on $\mathscr{F}_{R} X_{n}$. If $\iota(\partial D, F) \neq 0$ for some $F \in \mathscr{F}_{R} X_{n}, D \in \mathscr{F}_{R} X_{n+1}$, then $F \cap \partial D \neq \emptyset$ and hence $\left|\ell\left(\xi_{F}\right)-\ell\left(\xi_{D}\right)\right|$ remains uniformly bounded. Moreover, the number of summands in $\delta(D)$ is uniformly bounded for $D \in \mathscr{F}_{R} X_{n+1}$ because the boundary map $\delta$ on $\mathscr{F}_{R} X$ is bounded. If $f: X \rightarrow Y$ is a morphism, then for each $R \in \mathbb{N}$ there is $R^{\prime} \in \mathbb{N}$ such that $f_{*}: \operatorname{Cell} \bullet(X) \rightarrow \operatorname{Cell} \bullet(Y)$ maps $\operatorname{Cell} \bullet\left(\mathscr{F}_{R} X\right) \rightarrow \operatorname{Cell} \bullet\left(\mathscr{F}_{R^{\prime}} Y\right)$. If $\iota(f, D, E) \neq 0$ for two cells $D \in \mathscr{F}_{R} X_{n}, E \in \mathscr{F}_{R^{\prime}} Y_{n}$, then $f(D) \cap E \neq \emptyset$ and hence $\ell\left(\xi_{E}\right)=O\left(\ell\left(\xi_{D}\right)\right)$ because $f$ is quasi-Lipschitz and cells in $\mathscr{F}_{R} X$ are uniformly bounded. Since the sum $\sum_{E}|\iota(f, D, E)|$ is controlled uniformly for $D \in \mathscr{F}_{R} X_{n}$, we obtain that $f_{*}$ extends to a bounded linear map $f_{*}: \mathscr{T}$ Cellø $(X) \rightarrow \mathscr{T}$ Cell॰ $(Y)$. The argument for homotopies is identical.

Let $X$ be a discrete proper metric space and let $S(X)$ be the associated simplicial set. Everything is set up so that $\mathscr{T}$ Cell $|S(X)|$ is equal to $\mathscr{T} C_{\bullet}(X)$ if we turn $|S(X)|$ into a filtered metric $\mathrm{CW}$-complex as explained above. If $Y$ is any filtered metric CW-complex that is homotopy equivalent to $|S(X)|$ as such (it does not suffice to have a homotopy equivalence just as topological spaces!), then Lemma 14 yields a bornological homotopy equivalence between $\mathscr{T}$ Cell• $(Y)$ and $\mathscr{T} C \bullet \bullet(X)$. A necessary condition for this is that $Y$ should be contractible and $\mathscr{F}_{R} Y$ should be quasi-isometric to $X$, because $|S(X)|$ certainly has these properties and they are invariant under homotopy equivalence of filtered metric CW-complexes. Thus we consider a filtered metric CW-complex $Y$ together with a quasi-isometry $X \rightarrow \mathscr{F}_{R} Y$ for some $R \in \mathbb{N}$ in the following.

Recall that a $\mathrm{CW}$-complex $Y$ is contractible if and only if all its homotopy groups vanish. We need a controlled version of this criterion:

Definition 15. A filtered metric CW-complex $Y$ is uniformly contractible if for any $R \in \mathbb{N}$ there $R^{\prime} \in \mathbb{N}$ such that for any cellular map $f: S^{n} \rightarrow \mathscr{F}_{R} Y$ with $n \leq R,\|f\|_{n} \leq R$, and diameter of $f\left(S^{n}\right)$ at most $R$, there is an extension $\bar{f}: \bar{D}^{n+1} \rightarrow \mathscr{F}_{R^{\prime}} Y$ with $\|\bar{f}\|_{n+1} \leq R^{\prime}$, and diameter of $f\left(D^{n+1}\right)$ at most $R^{\prime}$. Here we use the cell decomposition with minimal number of cells for the $n$-sphere $S^{n}$ and the $n+1$-cell $D^{n+1}$ (that is, $D^{n+1}$ has 3 cells, of dimension $0, n$ and $n+1$, respectively, and the first two are those of $\left.S^{n} \subseteq D^{n+1}\right)$.

Theorem 16. Let $X$ be a discrete proper metric space and let $Y$ be a uniformly contractible, filtered metric $C W$-complex equipped with a quasi-isometry $X \rightarrow \mathscr{F}_{R} Y$ for some $R \in \mathbb{N}$. Then $Y$ is homotopy equivalent to $|S(X)|$ as a filtered metric $C W$ complex, and $\mathscr{T} C \bullet \bullet(X)$ is bornologically chain homotopy equivalent to $\mathscr{T}$ Cell• $(Y)$.

Proof. Let $Z$ be another filtered metric CW-complex with a quasi-isometric map $f: X \rightarrow \mathscr{F}_{R} Z$ for some $R \in \mathbb{N}$. We claim that there is a morphism of filtered metric CW-complexes $f^{\prime}: Z \rightarrow Y$ whose composition with $f$ is close to the given map $X \rightarrow Y$; moreover, any two such morphisms are homotopic. This universal property determines $Y$ uniquely up to homotopy equivalence of filtered metric $\mathrm{CW}$ complexes. It is easy to see that $|S(X)|$ is also uniformly contractible. Hence the homotopy equivalence of $|S(X)|$ and $Y$ follows from the above claim. The homotopy equivalence of $\mathscr{T}$ Cell $|S(X)|=\mathscr{T} C \bullet(X)$ then follows from Lemma 14

Thus it suffices to verify the universal property. Both existence and uniqueness are proven by induction on skeleta. The uniform contractibility guarantees the vanishing of the obstruction in the induction step. We only write down the details for the existence part. We have to construct functions $\rho_{n}: \mathbb{N} \rightarrow \mathbb{N}$ and cellular maps $f_{n}: Z^{(n)} \rightarrow Y$ with $\left.f_{n+1}\right|_{Z^{(n)}}=f_{n}$ for all $n \in \mathbb{N}$, such that $f_{n}\left(\mathscr{F}_{R} Z\right) \subseteq \mathscr{F}_{\rho_{n}(R)}(Y)$, the restriction of $f_{n}$ to $\mathscr{F}_{R} Z^{(n)}$ is Lipschitz with Lipschitz constant $\rho_{n}(R)$, and $\left\|\left.f_{n}\right|_{\mathscr{F}_{R}} Z\right\|_{n} \leq \rho_{n}(R)$, for all $R \in \mathbb{N}, n \in \mathbb{N}$. 
Since the given map $X \rightarrow \mathscr{F}_{R} Z \subseteq \mathscr{F}_{R^{\prime}} Z$ is a quasi-isometry for all $R^{\prime} \geq R$, we can find $f_{0}$ and $\rho_{0}$ with the required properties. In the induction step, we have to extend $f_{n}$ to $n+1$-cells. Such a cell $D$ is glued into $Z^{(n)}$ by a characteristic map $\chi_{D}: S^{n} \rightarrow Z^{(n)}$. We have to extend $f \circ \chi_{D}: S^{n} \rightarrow Y$ to a function $D^{n+1} \rightarrow Y$. If $D \in \mathscr{F}_{R} Z_{n+1}$, then $f \circ \chi_{D}$ takes values in $\mathscr{F}_{\rho_{n}(R)}(Y)$. It has Lipschitz constant $C(R, n) \cdot \rho_{n}(R)$, where $C(R, n)$ is the maximal diameter of $n+1$-cells in $\mathscr{F}_{R} Z$, and it has $n$th norm at most $\left\|\left.\delta\right|_{\mathscr{F}_{R} Z}\right\|_{n} \rho_{n}(R)$. Therefore, uniform contractibility allows us to find $\rho_{n+1}: \mathbb{N} \rightarrow \mathbb{N}$ and $f_{n+1}$ with the required properties.

Example 17. Let $G$ be a finitely generated discrete group and let $Y$ be a $G$-CWcomplex, equipped with the canonical filtered metric CW-complex structure defined in Example 13. Then $Y$ is uniformly contractible if and only if it is contractible. Of course, in this case we can get the assertion of Theorem 16 much more easily from Proposition 10 .

Example 18. Let $X$ be a discrete proper metric space and let $P_{R}(X), R \in \mathbb{N}$, be the associated Rips complexes. The union $P(X)=\bigcup P_{R}(X)$ is a filtered metric CW-complex if $X$ has bounded geometry. Otherwise the complexes $P_{R}(X)$ are not finite-dimensional and we have to modify the filtration slightly and take $P(X):=\bigcup P_{R}(X)^{(R)}$. The finite dimension of $\mathscr{F}_{R} Y$ is needed in the proof of Theorem 16. It is easy to check that $P(X)$ is uniformly contractible. Hence Theorem 16 yields that $|S(X)|$ and $P(X)$ are homotopy equivalent as filtered metric CW-complexes and that $\mathscr{T} C \bullet(X)$ and $\mathscr{T}$ Cell $(P(X))$ are bornologically homotopy equivalent.

If the metric space $X$ is hyperbolic, then $P_{R}(X)$ is uniformly contractible for sufficiently large $R$. Thus $|S(X)|$ is homotopy equivalent to $P_{R}(X)$ as a filtered metric CW-complex and $\mathscr{T} C \bullet(X) \cong \mathscr{T}$ Cell• $\left(P_{R}(X)\right)$. Since hyperbolic spaces have combings of linear growth, Theorem 5 implies that the complexes $\mathscr{S}$ Cell $\left(P_{R}(X)\right)$, $\mathscr{S}^{\omega}$ Cell. $\left(P_{R}(X)\right)$, and $\mathscr{O}$ Cell $\bullet\left(P_{R}(X)\right)$ are bornologically contractible.

\section{REFERENCES}

[1] Daniel J. Allcock and Stephen M. Gersten, A homological characterization of hyperbolic groups, Invent. Math. 135 (1999), no. 3, 723-742, doi: 10.1007/s002220050299. MR 1669272

[2] Martin R. Bridson, Combings of semidirect products and 3-manifold groups, Geom. Funct. Anal. 3 (1993), no. 3, 263-278, doi: 10.1007/BF01895689 MR 1215781

[3] Alain Connes and Henri Moscovici, Cyclic cohomology, the Novikov conjecture and hyperbolic groups, Topology 29 (1990), no. 3, 345-388, doi: 10.1016/0040-9383(90)90003-3 MR 1066176

[4] James W. Cannon, David B. A. Epstein, Derek F. Holt, Silvio V. F. Levy, Michael S. Paterson, and William P. Thurston, Word processing in groups, Jones and Bartlett Publishers, Boston, MA, 1992. MR 1161694

[5] Robert H. Gilman, Derek F. Holt, and Sarah Rees, Combing nilpotent and polycyclic groups, Internat. J. Algebra Comput. 9 (1999), no. 2, 135-155, doi: 10.1142/S0218196799000102 MR 1703070

[6] Henri Hogbe-Nlend, Complétion, tenseurs et nucléarité en bornologie, J. Math. Pures Appl. (9) 49 (1970), 193-288. MR 0279557

[7] _ Bornologies and functional analysis, North-Holland Publishing Co., Amsterdam, 1977. MR 0500064

[8] Ronghui Ji, Smooth dense subalgebras of reduced group $C^{*}$-algebras, Schwartz cohomology of groups, and cyclic cohomology, J. Funct. Anal. 107 (1992), no. 1, 1-33, doi: 10.1016/00221236(92)90098-4 MR 1165864

[9] Ralf Meyer, Bornological versus topological analysis in metrizable spaces, Banach algebras and their applications (Edmonton), Contemp. Math., vol. 363, Amer. Math. Soc., Providence, RI, 2004, pp. 249-278, doi: 10.1090/conm/363. MR 2097966

[10] _ Embeddings of derived categories of bornological modules (2004), eprint. arXiv: math.FA/0410596

[11] , Polynomial growth cohomology for combable groups, Oberwolfach reports 45 (2004), $2380-2384$. 
[12] _ Homological algebra for Schwartz algebras of reductive p-adic groups, Noncommutative geometry and number theory, Aspects Math., E37, Vieweg, Wiesbaden, 2006, pp. 263-300, doi: 10.1007/978-3-8348-0352-8_13 MR 2327309

[13] Igor Mineyev, $l_{1}$-homology of combable groups and 3-manifold groups, Internat. J. Algebra Comput. 12 (2002), no. 1-2, 341-355, doi: 10.1142/S0218196702000869 International Conference on Geometric and Combinatorial Methods in Group Theory and Semigroup Theory (Lincoln, NE, 2000). MR 1902370

Mathematisches Institut, Westfälische Wilhelms-Universität Münster, Einsteinstr. 62, 48149 Münster, Germany

E-mail address: rameyer@math.uni-muenster.de 